\title{
Does postural stability differ between adolescents with idiopathic scoliosis and typically developed? A systematic literature review and meta-analysis
}

\author{
Marlene Dufvenberg $^{1 *}$ (D, Fisayo Adeyemi ${ }^{2}$, Isabelle Rajendran ${ }^{2}$, Birgitta Öberg ${ }^{1}$ and Allan Abbott ${ }^{1,2}$
}

\begin{abstract}
Background: Postural stability deficits have been proposed to influence the onset and progression of adolescent idiopathic scoliosis (AIS). This study aimed to systematically identify, critically evaluate and meta-analyse studies assessing postural stability during unperturbed stance with posturography in AIS compared to typically developed adolescents.
\end{abstract}

Methods: Studies from four electronic databases (PubMed, Scopus, CINAHL, PEDro) were searched and case-control methodological quality assessed using a risk-of-bias assessment tool and a posturography methodological quality checklist. Pooled data regarding centre of pressure (COP) parameters such as sway area, Mediolateral (ML) and Anteroposterior (AP) position and range were compared for AIS and typically developed adolescents using Cohen's $d$ effect size (ES) and homogeneity estimates.

Results: Eighteen studies for quality analysis and 9 of these for meta-analysis were identified from 971 records. Riskof-bias assessment identified 6 high, 10 moderate and 2 low risk-of-bias studies. The posturography methodological quality checklist identified 4 low, 7 moderate and 7 high-quality studies. Meta-analysis was performed for sway area whereas ML and AP are presented in three different meta-analyses due to divergent measurement units used in the studies: ML position 1 (MLP1), ML position 2 (MLP2) and ML range (MLR); AP position 1 (APP1), AP position 2 (APP2) and AP range (APR). Cohen's d showed a medium ES difference in sway area 0.65, 95\% Cl (0.49-0.63), whereas ML showed no (MLP1, MLP2) and large (MLR) ES differences; MLP1 0.15, 95\% CI (0.08-0.22); MLP2 0.14, 95\% Cl (0.08-0.19); and MLR 0.94, 95\% Cl (0.83-1.04). Cohen's d for AP showed small ES (APP1) and large ES difference (APP2 and APR); APP1 0.43, 95\% Cl (0.31-0.54); APP2 0.85, 95\% Cl (0.72-0.97); and APR 0.98, 95\% Cl (0.87-1.09). Cochran's Q and Higgins $P$ showed homogeneity between studies.

Conclusions: There is moderate quality evidence for decreased postural stability in AIS measured as COP parameters sway area, ML and AP range with a positional shift posteriorly in the sagittal plane. The findings support studying postural stability in early stage AIS and also prospectively identify cause and effect of the curvature as well as effectiveness of postural control interventions in the prevention of scoliosis progression.

Keywords: Adolescent idiopathic scoliosis, Postural balance, Postural control, Postural stability, Posturography, Force plate, Centre of pressure, Sway area, Anteroposterior, Mediolateral

\footnotetext{
* Correspondence: marlene.dufvenberg@liu.se

${ }^{1}$ Department of Medical and Health Sciences, Division of Physiotherapy,

Linkoping University, 58183 Linkoping, Sweden

Full list of author information is available at the end of the article
}

(c) The Author(s). 2018 Open Access This article is distributed under the terms of the Creative Commons Attribution 4.0 International License (http://creativecommons.org/licenses/by/4.0/), which permits unrestricted use, distribution, and reproduction in any medium, provided you give appropriate credit to the original author(s) and the source, provide a link to the Creative Commons license, and indicate if changes were made. The Creative Commons Public Domain Dedication waiver (http://creativecommons.org/publicdomain/zero/1.0/) applies to the data made available in this article, unless otherwise stated. 


\section{Background}

Postural control involves the body's position in space for dual purposes of stability and orientation [1]. Postural stability is often described as the ability to control the centre of body mass (COM) relative to the base of support (BOS) [1] while postural orientation is the ability to maintain an appropriate relationship between the body segments and between the body and the environment for a task [2-4]. COM is often used interchangeably with the term centre of gravity (COG) [5]. Postural control in fairly predictable and non-challenging conditions, e.g. standing quietly is defined as steady-state balance [1]. Postural sway always occurs during quiet standing because of our vertical posture which is inherently unstable due to our relatively high COM $[3,6]$. Postural control is a complex skill based on the interaction of multiple dynamic sensorimotor and cognitive systems to maintain postural stability under static and dynamic conditions $[1,2,7]$. Pathology to any of the underlying systems will result in different, context-specific postural deficits [7].

All neuromuscular disorders that act on the growing body, particularly during the rapid pubertal growth period, can lead to the development of scoliosis [8]. Adolescent idiopathic scoliosis (AIS) is a three-dimensional deformity of the spine and trunk with a lateral deviation of $\geq 10^{\circ}$ which can occur between the ages of 10 and 17. It is the most common cause of spinal deformity in otherwise healthy adolescents [9-11]. AIS affects 0.47 to $5.2 \%$ of the general population, with an apparent gender dominance, rising with age and severity, with a female to male ratio from $1.5: 1$ to $10: 1$ [10-12]. This condition is associated with a higher incidence of back pain and discontent with body image and can in severe cases lead to pulmonary functional deficits [13].

The pathogenesis of AIS remains unknown but is considered to be multifactorial [14-16]. A disharmony between autonomic and somatic nervous systems has been hypothesised to cause changed regulation of somatosensory input and motor output $[8,17]$. These changes may cause postural stability deficits affecting the onset and curve progression [18]. Previous studies on changes in postural stability deficits for individuals with AIS compared to typically developed adolescents (CON) have shown inconsistent results from significant differences [19] to no differences in balance tasks [20, 21].

There are a variety of methods to assess postural control during different conditions and ages [22-24]. Posturography, bipedal static task on force plate (force platform), is the most used devise to provide an indirect assessment of changes in postural sway to gain a better understanding of quiet standing balance [22, 24, 25]. Force plate measures ground reaction forces (GRF) that represent the sum of the pressure distributed under the foot. Centre of pressure (COP) refers to the point at which the pressure would be concentrated $[5,26]$. Postural stability can thus be quantified using COP parameters derived from GRF to assess alterations in balance $[22,25,26]$. Posturography has been used to determine if postural stability is changed in AIS and if type and location of scoliosis affect progression [27].

Various COP parameters calculated as sway area, mediolateral (ML), and anteroposterior (AP) change of position and range have been derived from COP data [22, 24, 26]. Sway area refers to body oscillations, often described as a $95 \%$ ellipse area which is expected to enclose approximately $95 \%$ of the points on the COP path [22, 24]. ML and AP assessed as position defines an object's location [22] whereas range is the maximum distance between any two points on the COP path relative to a baseline value or axis [24]. Mean amplitude of COP is an average value over all data points collected in a trial and is a representative measure of postural control [24, 26].The movement of COP in the positive direction, according to right-hand coordinate system, is towards the right in the frontal plane whereas the AP direction indicates a forward displacement in the sagittal plane from the central body position [28]. Increased values are an indication of decreased postural stability $[22,26]$. The reliability of COP measures have been criticised but can be used as a reliable tool for investigating general postural stability under specific conditions [29, 30].

The purpose of this review was to identify, critically evaluate and meta-analyse studies assessing postural stability during unperturbed stance with posturography in AIS compared to CON. We hypothesised that AIS would have decreased postural stability compared to typically developed adolescents measured as COP parameters sway area, ML and AP position and/or range.

\section{Methods}

\section{Data sources and searches}

Studies published until the end of 2016 was retrieved from a search of four electronic databases: PubMed, Scopus, CINAHL and PEDro in April-May 2017 to identify eligible studies. The authors in consultation with an academic librarian designed the search strategy to identify relevant studies comparing postural stability within AIS compared to a control group with typically developed adolescents (CON). The following main search terms were used: ("scoliosis" AND "adolescent" AND "postural AND sway" OR "postural AND stability" OR "postural" AND "control" OR "postural” AND "balance"), appropriate to each database (Table 1). A reference list search was carried out on included full-text studies.

\section{Screening and selection}

The inclusion criteria were as follows: (1) adolescents aged 10-18 years; (2) use of force plate to measure postural stability during stance; (3) inclusion of one or more reported parameters: mean sway area, mean position and/or range in ML and AP direction; (4) AIS and typically developed 
Table 1 Overview of search strategy and retrieved studies

\begin{tabular}{|c|c|c|c|c|c|c|}
\hline $\begin{array}{l}\text { Database } \\
\text { Date of } \\
\text { search } \\
\mathrm{dd} / \mathrm{mm} / \mathrm{yy}\end{array}$ & $\begin{array}{l}\text { Search period } \\
\text { included } \\
\mathrm{dd} / \mathrm{mm} / \mathrm{yy}\end{array}$ & Search terms & $\begin{array}{l}\text { Total } \\
\text { number }\end{array}$ & $\begin{array}{l}\text { Number from } \\
\text { title and abstract }\end{array}$ & Duplicated & $\begin{array}{l}\text { New } \\
\text { studies }\end{array}$ \\
\hline $\begin{array}{l}\text { Scopus } \\
18 / 04 / 17\end{array}$ & $31 / 12 / 16$ & $\begin{array}{l}\text { ((TITLE-ABS-KEY(idiopathic AND scoliosis) OR } \\
\text { (idiopathic AND scolioses) OR AIS } \\
\text { OR scoliosis OR (spinal AND deformity) } \\
\text { OR (spinal AND deformities) OR } \\
\text { scoliotic OR scolioses OR } \\
\text { (spinal AND curvature) OR } \\
\text { (spinal AND curvatures))) } \\
\text { AND (TITLE-ABS-KEY ((postural AND sway) } \\
\text { OR (postural AND stability) OR } \\
\text { (postural AND function) OR } \\
\text { (postural AND control) OR } \\
\text { (postural AND behaviour) OR } \\
\text { (postural AND behavior) OR } \\
\text { (postural AND performance) OR } \\
\text { (postural AND regulation) OR } \\
\text { (postural AND strategy) OR } \\
\text { (postural AND strategies) OR } \\
\text { (postural AND dysfunction) OR } \\
\text { (postural AND dysfunctionality) OR } \\
\text { (body AND balance) OR (body AND sway) } \\
\text { OR (postural AND control AND system) OR } \\
\text { (postural AND balance) OR } \\
\text { (body AND equilibrium)))) } \\
\text { AND (TITLE-ABS-KEY } \\
\text { ((adolescen* OR youth* OR teenager*))) }\end{array}$ & 458 & 23 & - & 0 \\
\hline $\begin{array}{l}\text { PubMed } \\
\text { 06/04/17 }\end{array}$ & 23/09/16 & $\begin{array}{l}\text { (((((((((Scoliosis OR spinal curvatures[MeSH Terms]))) OR } \\
((\text { Idiopathic Scoliosis OR AIS OR Scoliosis OR } \\
\text { spinal deformity OR scoliotic OR scolioses OR } \\
\text { spinal curvatures)))) AND } \\
((((\text { Adolescent OR Adolescence OR youth OR } \\
\text { teenager[MeSH Terms]))) OR ((Adolescent OR } \\
\text { Adolescence OR youth OR teenager))))) AND } \\
((((\text { Postural sway OR postural stability OR } \\
\text { postural function OR postural control OR } \\
\text { postural behaviour OR postural performance OR } \\
\text { postural regulation OR postural strategy OR } \\
\text { postural dysfunction OR body balance OR } \\
\text { body sway OR postural control system OR } \\
\text { postural balance OR body equilibrium))) } \\
\text { OR postural balance[MeSH Terms]])) }\end{array}$ & 396 & 16 & $\begin{array}{l}300 \\
249 \\
\text { Scopus, } \\
56 \text { CINAHL } \\
1 \text { PEDro }\end{array}$ & 0 \\
\hline $\begin{array}{l}\text { CINAHL } \\
18 / 05 / 17\end{array}$ & 23/09/16 & $\begin{array}{l}\text { (Idiopathic Scoliosis OR AIS OR Scoliosis OR } \\
\text { spinal deformity OR scoliotic OR scolioses OR } \\
\text { spinal curvatures) AND (Postural sway OR } \\
\text { postural stability OR postural function OR } \\
\text { postural control OR postural behaviour OR } \\
\text { postural performance OR postural regulation OR } \\
\text { postural strategy OR postural dysfunction OR } \\
\text { body balance OR body sway OR } \\
\text { postural control system OR } \\
\text { postural balance OR body equilibrium) AND } \\
\text { (Adolescent OR Adolescence OR } \\
\text { youth OR teenager) }\end{array}$ & 81 & 1 & $\begin{array}{l}107 \\
51 \text { Scopus } \\
56 \text { PubMed } \\
\text { 0 PEDro }\end{array}$ & 0 \\
\hline $\begin{array}{l}\text { PEDro } \\
18 / 05 / 17\end{array}$ & $31 / 12 / 16$ & Scoliosis and Clinical trial & 36 & 0 & 1 PubMed & 0 \\
\hline \multicolumn{2}{|c|}{ All databases } & & 971 & & 357 & \\
\hline \multicolumn{2}{|c|}{ After duplicates } & & 614 & & & \\
\hline \multicolumn{3}{|c|}{ Retrieved for full text } & & 23 & & 0 \\
\hline
\end{tabular}

adolescents; (5) case-control studies; and (6) studies published in English. The exclusion criteria were as follows: (1) non-idiopathic scoliosis; (2) non-healthy subjects (other neuromuscular, neurological, congenital or trauma-related co-morbidities); (3) patients who have undergone scoliosis correctional surgery or in brace; (4) 


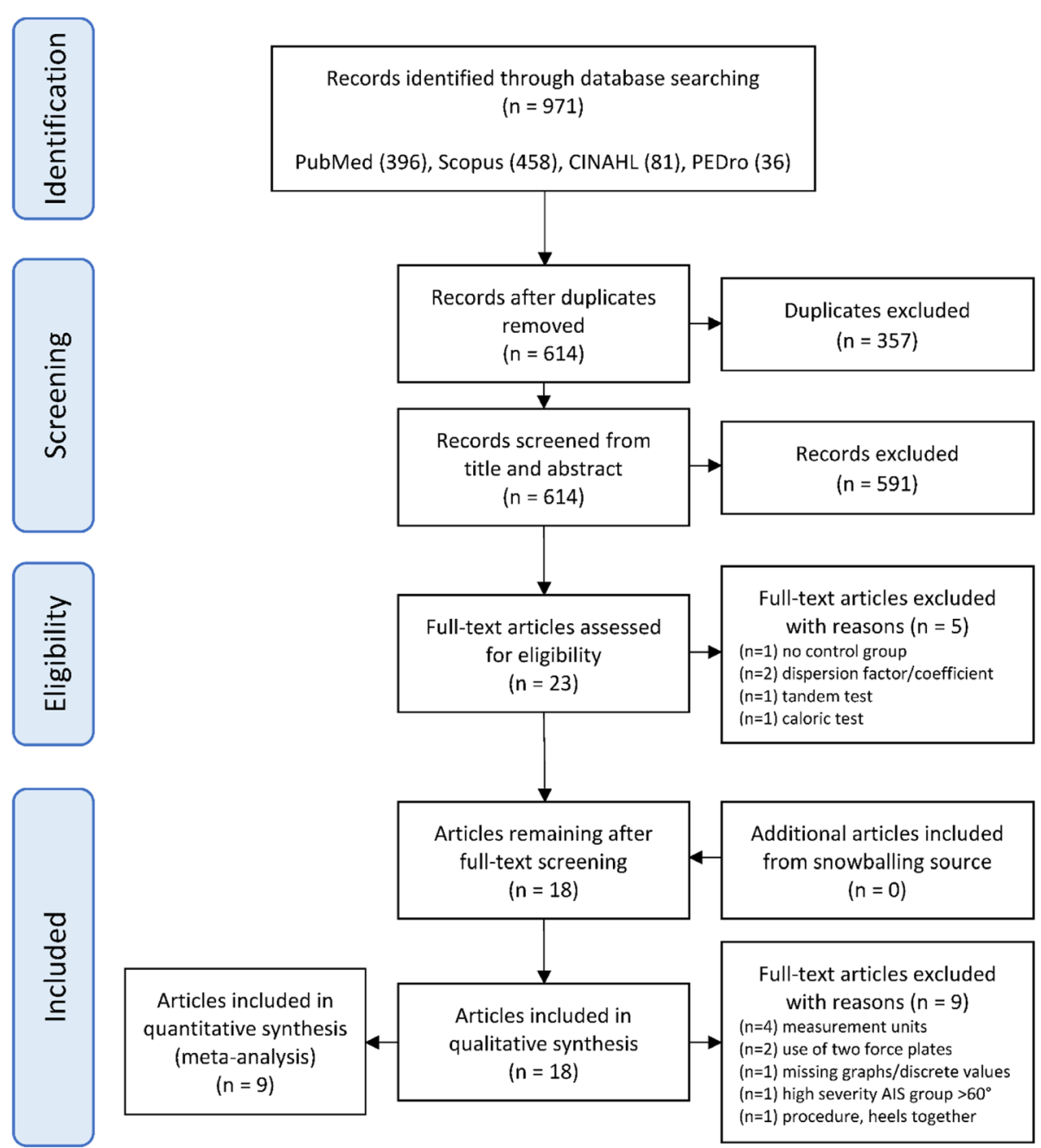

Fig. 1 PRISMA flow diagram describing selection process for included studies

Table 2 Risk-of-bias assessment tool for case-control methodology

\begin{tabular}{|c|c|}
\hline Item & Scoring \\
\hline \multicolumn{2}{|l|}{ Selection: } \\
\hline 1. Is the control group representative for normal adolescents? & $\begin{array}{l}1=\text { Community control; } 0=\text { Hospital controls; } \\
0=\text { No description of source }\end{array}$ \\
\hline 2. Was other pathology excluded that possibly influences the outcome? & $1=$ Yes; $0=$ No or no description \\
\hline \multicolumn{2}{|l|}{ Comparability: } \\
\hline $\begin{array}{l}\text { 3. Were the same in- and exclusion criteria (except for the spinal deformity) used for AIS and } \\
\text { healthy adolescents? }\end{array}$ & $1=$ Yes; $0=$ No or no description \\
\hline \multicolumn{2}{|l|}{ Exposure/outcome: } \\
\hline 4. Were the observers blinded to AIS/healthy adolescent status? & $1=$ Yes; $0=$ No or not documented \\
\hline $\begin{array}{l}\text { 5. Was the data collection performed in the same standardised way for AIS cases and healthy } \\
\text { adolescents? }\end{array}$ & $1=$ Yes; $0=$ No or not documented \\
\hline 6. ${ }^{a}$ Was the primary outcome parameter for AIS cases and healthy adolescents available? & $\begin{array}{l}1=\text { Available for }>90 \% \text { of AIS and healthy } \\
\text { adolescents; } \\
0=\text { Available for }<90 \% \text { of AIS or healthy } \\
\text { adolescents }\end{array}$ \\
\hline
\end{tabular}


an absence of CON; and (5) measurements during perturbed stance. Further exclusion criteria for meta-analysis were (6) divergent measurement units or discrete values and graphs missing, (7) use of two force plates, (8) procedure with feet or heels together, (9) and high severity scoliosis group, Cobb $>60^{\circ}$.

The study followed the PRISMA reporting guidelines and a flow diagram was used to document the study screening and selection process [31] (Fig. 1). From the initial studies, identified duplicates were removed. Two assessors (M.D., F.A.) manually screened the titles and abstracts for relevance and likelihood of meeting the inclusion and exclusion criteria. Three appraisers (M.D., F.A., I.R.) evaluated remaining studies using the inclusion and exclusion criteria to yield studies for the qualitative and quantitative synthesis.

\section{Critical appraisal}

Methodological quality was assessed using two critical appraisal tools. A risk-of-bias assessment tool for case-control methodology (Table 2) was chosen due to its population specificity [32]. A six-item scoring system was performed for description and validity of key information to facilitate categorisation of the risk-of-bias impact on the quality of studies, one point for each criterion with minimal score of 0 and a maximal score of 6 possible. Cut off values suggested by Schlösser et al. [32] were not used for exclusion of studies. Included studies were instead ranked according to the following 0-2 as high, 3-4 moderate, and 5-6 being a low risk of bias. The posturography methodological quality checklist (Table 3) was selected to consider internal and external validity and to identify differences that may explain heterogeneity between studies [33]. The total number of items with positive points were divided by the total number of items and multiplied by 100 to produce a quality score for each domain. An overall measure of quality was found by averaging each domain score. Studies were further ranked according to their total score: $\leq$ 49 = low, $\geq 50$ = moderate and $\geq 80$ = high quality. Three independent blinded appraisers undertook the assessment (M.D., F.A., I.R.). For any disagreements between appraisers' discussions, a fourth reviewer (A.A.) participated to reach consensus.

\section{Data extraction and synthesis}

Key data regarding the study settings, participant demographics, study protocol, COP parameters, results and

Table 3 The posturography methodological quality checklist

\begin{tabular}{|c|c|c|}
\hline Item & Description & Scoring \\
\hline \multicolumn{3}{|c|}{ Internal validity } \\
\hline 1 & Indicates reliability of outcome measures & $\begin{array}{l}\text { A positive point was assigned if a minimum } \\
\text { sampling duration of } 90 \mathrm{~s} \text { and/or } 3-5 \text { reps was used }\end{array}$ \\
\hline 2 & Clear presentation of balance assessment & $\begin{array}{l}\text { A positive point was assigned if replication of } \\
\text { the experiment is possible based on the } \\
\text { information in the article }\end{array}$ \\
\hline \multirow[t]{5}{*}{3} & Correction for confounding effect on dependent variables & $\begin{array}{l}\text { Positive points were assigned if confounders } \\
\text { were taken into account, or appropriate matching } \\
\text { on these variables was performed }\end{array}$ \\
\hline & & 3a Study controls for age \\
\hline & & 3b Study controls for gender \\
\hline & & 3c Study controls for height \\
\hline & & 3d Study controls for body mass \\
\hline \multicolumn{3}{|c|}{ Statistical validity } \\
\hline 4 & The use of appropriate statistical tests & $\begin{array}{l}\text { A positive point was assigned if appropriate } \\
\text { tests were used to assess differences in balance }\end{array}$ \\
\hline 5 & Adequate sample size & $\begin{array}{l}\text { A positive point was assigned if a minimum } \\
\text { of } 20 \text { subjects per group were included }\end{array}$ \\
\hline \multicolumn{3}{|c|}{ External validity } \\
\hline \multirow[t]{6}{*}{6} & Sufficient information about the subject's characteristics & Positive if information was provided about \\
\hline & & 6a Adequate information regarding age \\
\hline & & 6b Adequate information regarding gender \\
\hline & & 6c Adequate information regarding height \\
\hline & & $6 \mathrm{~d}$ Adequate information regarding body mass \\
\hline & & $\begin{array}{l}\text { 6e Adequate information regarding severity of } \\
\text { curve (Cobb angle) }\end{array}$ \\
\hline
\end{tabular}


Table 4 Overview of risk-of-bias assessments of all included studies and total score

\begin{tabular}{|c|c|c|c|c|c|c|c|}
\hline \multirow[t]{2}{*}{ Author/year } & \multicolumn{2}{|c|}{ Risk-of-bias selection } & \multirow{2}{*}{$\begin{array}{l}\text { Risk-of-bias comparability } \\
\text { Item } 3\end{array}$} & \multicolumn{3}{|c|}{ Risk-of-bias exposure/outcome } & \multirow{2}{*}{$\begin{array}{l}\text { Total } \\
\text { score } \\
0-6 \\
\text { points }\end{array}$} \\
\hline & Item 1 & Item 2 & & Item 4 & Item 5 & Item 6 & \\
\hline Sahli et al. 2013 [42] & 1 & 1 & 1 & 0 & 1 & 1 & 5 \\
\hline Park et al. 2013 [43] & 0 & 1 & 0 & 0 & 1 & 1 & 3 \\
\hline De Santiago et al. 2013 [44] & 0 & 0 & 0 & 0 & 1 & 1 & 2 \\
\hline Stylianides et al. 2013 [45] & 0 & 1 & 0 & 0 & 1 & 1 & 3 \\
\hline Gruber et al. 2011 [46] & 0 & 1 & 1 & 0 & 1 & 1 & 3 \\
\hline Dalleau et al. 2011 [47] & 0 & 1 & 1 & 0 & 1 & 1 & 4 \\
\hline Valles et al. 2009 [48] & 0 & 1 & 0 & 0 & 1 & 0 & 2 \\
\hline Beaulieu et al. 2009 [49] & 1 & 1 & 1 & 0 & 1 & 1 & 5 \\
\hline Zabjek et al. 2008 [50] & 0 & 0 & 0 & 0 & 1 & 1 & 2 \\
\hline Dalleau et al. 2007 [51] & 0 & 1 & 0 & 0 & 1 & 1 & 3 \\
\hline Simoneau, et al. 2006 [52] & 0 & 1 & 0 & 0 & 1 & 1 & 3 \\
\hline Chow et al. 2006 [53] & 1 & 1 & 0 & 0 & 1 & 1 & 4 \\
\hline Simoneau et al. 2006 [54] & 0 & 0 & 0 & 0 & 1 & 1 & 2 \\
\hline Allard et al. 2004 [55] & 1 & 1 & 0 & 0 & 1 & 1 & 4 \\
\hline Silferi et al. 2004 [56] & 0 & 0 & 0 & 0 & 1 & 1 & 2 \\
\hline Nault et al. 2002 [57] & 0 & 0 & 0 & 0 & 1 & 1 & 2 \\
\hline Chen et al. 1998 [58] & 0 & 1 & 0 & 0 & 1 & 1 & 3 \\
\hline Sahlstrand et al. 1978 [59] & 1 & 1 & 0 & 0 & 1 & 1 & 4 \\
\hline
\end{tabular}

Item 1: Is the control group representative for normal adolescents? $1=$ Community control; $0=$ Hospital controls/No description of source. Item 2: Was other pathology excluded that possibly influences the outcome? $1=$ Yes; $0=$ No or no description. Item 3: Were the same in- and exclusion criteria (except for the spinal deformity) used for AIS and healthy adolescents? $1=$ Yes; $0=$ No or no description. Item 4: Were the observers blinded to AIS/healthy adolescent status $1=$ Yes; $0=$ No or not documented. Item 5: Was the data collection performed in the same standardised way for AIS cases and healthy adolescents? $1=$ Yes; $0=$ No or not documented. Item 6: Was the primary outcome parameter for AIS cases and healthy adolescents available? $1=$ Available for $>90 \%$ of AIS and healthy adolescents, $0=$ available for $<90 \%$ of AIS or healthy adolescents. Total score: Ranked according to total score; $0-2$ high, 3-4 moderate and 5-6 low risk of bias

conclusions were extracted and tabulated. If discrete values were not reported, estimated values were extracted from graphs or figures. A critical qualitative synthesis was conducted with a consideration of risk-of-bias assessment and posturography methodology (Tables 2 and 3).

To evaluate the quantitative differences in postural stability in AIS compared with CON, meta-analysis was conducted. Increased values for one of the groups expressed decreased postural stability. Means and standard deviations (SD) for posturography data were collected from each study; if multiple AIS subgroups were presented, combined means were calculated. All measures were converted to square millimetres and millimetres. Confidence interval (CI) and standard error/of measurements (SE and SEM) were converted to SD (two-tailed, 95\% CI). Effect sizes (ES) (Cohen's d) [34] for COP data collected in each study was calculated using an online calculator $[35,36]$. The statistical calculations for meta-analysis and ES were performed using Microsoft Office Excel meta-analysis package [37]. The meta-analysis package included a built-in random-effects model considering ES and how homogeneous the data was from the different studies. As a result, the overall (pooled) ES for these studies and a confidence interval (CI) of 95\% could be calculated [37]. According to Cohen [34], $d=0.20$ is considered as a small, $d=0.50$ medium and $d=0.80$ a large ES difference.

Cochran's Q and Higgins's $I^{2}$ statistics were calculated to assess homogeneity across studies using the random-effects model [38, 39]. Cochran's Q was compared to a chi-square critical values table $\left(\chi^{2}\right)$, alpha level $p<0.05$, where degrees of freedom are the number of studies in the analysis minus one. If the Cochran's $Q$ value is under the chi-square value, we accept the null hypothesis that all studies are homogeneous [38]. Higgin's $I^{2}$ is an estimate of the percentage of total variability that can be attributed to the variation between studies [40]. Thus, percentages of Higgins's $I^{2}$ around 25\% $\left(I^{2}=25\right), 50 \%\left(I^{2}=\right.$ $50)$ and $75 \%\left(I^{2}=75\right)$ were classified as low, medium and high heterogeneity, respectively. In this review, $I^{2}$ below $25 \%$ suggests homogeneity $[38,41]$.

In this review, seven meta-analyses were calculated from COP parameters. One represents sway area while ML and AP are each presented as three different meta-analyses due to divergent measurement units used in the included studies: ML position 1 (MLP1), ML position 2 (MLP2) 
Table 5 Overview of posturography methodological quality assessments of all included studies and total score

\begin{tabular}{|c|c|c|c|c|c|c|c|c|c|c|c|c|c|c|c|c|c|}
\hline \multirow[t]{2}{*}{ Author/year } & \multicolumn{6}{|c|}{ Internal validity } & \multirow[t]{2}{*}{ Score } & \multicolumn{2}{|c|}{ Statistical validity } & \multirow[t]{2}{*}{ Score } & \multicolumn{5}{|c|}{ External validity } & \multirow[t]{2}{*}{ Score } & \multirow{2}{*}{$\begin{array}{l}\text { Total } \\
\text { score }\end{array}$} \\
\hline & 1 & 2 & $3 a$ & $3 b$ & $3 c$ & $3 d$ & & 4 & 5 & & $6 a$ & $6 b$ & $6 c$ & $6 d$ & $6 e$ & & \\
\hline Sahli et al. 2013 [42] & + & + & + & - & + & + & 83 & + & - & 50 & + & - & + & + & + & 80 & 71 \\
\hline Park et al. 2013 [43] & - & - & + & - & + & - & 33 & + & - & 50 & + & - & + & + & + & 80 & 54 \\
\hline de Santiago et al. 2013 [44] & + & + & + & + & - & - & 67 & + & - & 50 & + & + & + & + & + & 100 & 66 \\
\hline Stylianides et al. 2013 [45] & + & + & + & + & + & + & 100 & + & + & 100 & + & + & + & + & + & 100 & 100 \\
\hline Gruber et al. 2011 [46] & + & - & + & + & - & + & 66 & + & - & 50 & + & + & + & + & + & 100 & 72 \\
\hline Dalleau et al. 2011 [47] & + & + & + & + & + & + & 100 & + & + & 100 & + & + & + & + & + & 100 & 100 \\
\hline Valles et al. 2009 [48] & + & + & - & - & - & - & 33 & + & - & 50 & - & - & - & - & - & 0 & 28 \\
\hline Beaulieu et al. 2009 [49] & + & + & + & + & + & + & 100 & + & + & 100 & + & + & + & + & + & 100 & 100 \\
\hline Zabjek et al. 2008 [50] & + & - & + & + & + & + & 83 & + & - & 50 & + & + & + & + & + & 100 & 78 \\
\hline Dalleau et al. 2007 [51] & + & + & + & + & + & + & 100 & + & + & 100 & + & + & + & + & + & 100 & 100 \\
\hline Simoneau et al. 2006 [52] & + & + & - & - & - & - & 33 & + & - & 50 & + & + & - & - & + & 60 & 48 \\
\hline Chow et al. 2006 [53] & + & + & + & + & + & + & 100 & + & + & 100 & + & + & + & + & + & 100 & 100 \\
\hline Simoneau et al. 2006 [54] & + & + & + & - & - & - & 50 & + & - & 50 & + & + & - & - & + & 60 & 53 \\
\hline Allard et al. 2004 [55] & + & + & + & + & + & + & 100 & + & + & 100 & + & + & + & + & + & 100 & 100 \\
\hline Silferi et al. 2004 [56] & + & + & - & - & - & - & 33 & + & - & 50 & + & + & - & - & - & 40 & 41 \\
\hline Nault et al. 2002 [57] & + & + & + & + & + & + & 100 & + & + & 100 & + & + & + & + & + & 100 & 100 \\
\hline Chen et al. 1998 [58] & - & + & - & - & - & - & 17 & - & - & 50 & + & + & - & - & + & 60 & 42 \\
\hline Sahlstrand et al. 1978 [59] & + & + & - & + & - & - & 50 & + & + & 100 & + & + & - & - & - & 40 & 63 \\
\hline
\end{tabular}

Items indicate: Internal validity (1) reliability of outcome measures; (2) clear presentation of balance assessment; (3a) study controls for age; (3b) study controls for gender; (3c) study controls for height; (3d) study controls for body mass; Statistical validity (4) use of appropriate statistical tests; (5) adequate sample size; External validity (6a) Adequate information regarding age; (6b) adequate information regarding gender; (6c) adequate information regarding height; (6d) adequate information regarding body mass; $(6 \mathrm{e})$ adequate information regarding severity of curve (Cobb angle)

and ML range (MLR); AP position 1 (APP1), AP position 2 (APP2) and AP range (APR), where APP2 describes the change in position from a location from the back of the heels and leaning forward in opposite to APP1 where the location is estimated from the centre point of support and backwards. The three different categorisations were performed likewise for MLP1 and MLP 2 to minimise the risk of heterogeneity in the meta-analyses.

\section{Results}

\section{Literature review}

Electronic database searches yielded 971 potential studies (Table 1). After title and abstract review and removal of duplicates, 23 studies were retrieved in full text (Fig. 1). The full-text review excluded 5 studies due to the following reasons: no $\mathrm{CON}$, dispersion factor or coefficient as parameter, during caloric, and during tandem test. This resulted in 18 studies for inclusion in the synthesis component of the study from the most recent to the oldest published [42-59]. A search from reference lists yielded no additional studies (Fig. 1).

\section{Critical appraisal of methodological quality}

The scoring of methodological quality for each study using a risk-of-bias assessment tool is outlined in Table 4.
After evaluating 18 studies, 6 [44, 48, 50, 54, 56, 57] showed a high risk of bias (total score 2), 10 [43, 45-47, $51-53,55,58,59$ ] showed a moderate risk of bias (total score 3-4) and two [42, 49] showed a low risk of bias (total score 5). Nil studies scored the lowest risk of bias (total score 6) or the highest risk of bias (total score 0) possible. Four out of 18 studies [42, 46, 47, 49] met the criteria of the same inclusion and exclusion criteria for $\mathrm{CON}$ and AIS.

Table 5 outlines the results of the posturography methodological quality checklist. The appraisers identified 4 low (total score $\leq 49$ ) $[48,52,56,58], 7$ moderate (total score $\geq 50)[42-44,46,50,54,59]$ and 7 high-quality studies (total score $>80$ ) $[45,47,49,51,53,55,57]$.

\section{Participants}

The population, setting of recruitment, anthropometric measures, and scoliosis characteristics such as Cobb angle and primary curve type for AIS participants are summarised in Table 6. This review included a total of 954 participants (402 CON; 552 AIS) with mean age of $13.9 \pm 1.8$ for CON and $13.9 \pm 1.9$ for AIS. A total of 758 females (345 CON; 413 AIS) and 23 males (10 CON; 13 AIS) were included in the studies, and for the remaining 175 participants, gender was not reported [42, 43, 48]. A total of 13 studies reported the AIS 
Table 6 Participant characteristics of CON and AIS from all included studies

\begin{tabular}{|c|c|c|c|c|c|c|c|c|}
\hline \multirow{2}{*}{$\begin{array}{l}\text { Author } \\
\text { Year }\end{array}$} & \multirow{2}{*}{$\begin{array}{l}\text { Country and } \\
\text { setting of participant } \\
\text { recruitment }\end{array}$} & \multicolumn{3}{|l|}{$\mathrm{CON}$} & \multicolumn{4}{|l|}{ AIS } \\
\hline & & $\begin{array}{l}N=\text { Sample } \\
\text { size, gender } \\
=0 \text { + }{ }^{N}\end{array}$ & $\begin{array}{l}\text { Age } \\
\pm \mathrm{SD} \\
\text { (years) }\end{array}$ & $\begin{array}{l}\text { Height } \pm \text { SD } \\
(\mathrm{cm}) \text {, weight } \\
\pm \mathrm{SD}(\mathrm{kg})\end{array}$ & $\begin{array}{l}N=\text { Sample } \\
\text { size, } \\
\text { gender } \\
=+: 0^{\lambda}\end{array}$ & $\begin{array}{l}\text { Cobb }\left({ }^{\circ}\right) \text { : range and/or mean } \\
\pm \mathrm{SD} \text {, primary curve type }\end{array}$ & $\begin{array}{l}\text { Age } \pm \\
\text { SD } \\
\text { (years) }\end{array}$ & $\begin{array}{l}\text { Height } \pm \text { SD }(\mathrm{cm}) \\
\text { weight } \pm \text { SD }(\mathrm{kg})\end{array}$ \\
\hline $\begin{array}{l}\text { Sahli et al. } \\
2013 \text { [42] }\end{array}$ & $\begin{array}{l}\text { CON: Community, } \\
\text { Tunisia } \\
\text { AIS: Hospital, } \\
\text { Tunisia }\end{array}$ & $\begin{array}{l}N=12 \\
\text { gender }= \\
\text { NR }\end{array}$ & $\begin{array}{l}13.2 \\
\pm 1.6\end{array}$ & $\begin{array}{l}159.0 \pm 8.0 \\
45.3 \pm 6.6\end{array}$ & $\begin{array}{l}N=14 \\
\text { gender }= \\
13: 1\end{array}$ & $\begin{array}{l}\text { Range } 10^{\circ}-28^{\circ}, N=10 \text { Right } \\
\text { thoracic, } N=4 \text { left lumbar }\end{array}$ & $\begin{array}{l}14.2 \pm \\
1.6\end{array}$ & $160 \pm 9.0,51.8 \pm 8.8$ \\
\hline $\begin{array}{l}\text { Park et al. } \\
2013 \text { [43] }\end{array}$ & $\begin{array}{l}\text { CON: NR } \\
\text { AIS: Hospital, } \\
\text { South Korea }\end{array}$ & $\begin{array}{l}N=15 \\
\text { gender }= \\
\text { NR }\end{array}$ & $\begin{array}{l}14.7 \\
\pm 1.7\end{array}$ & $\begin{array}{l}160 \pm 6.0 \\
65.2 \pm 10.4\end{array}$ & $\begin{array}{l}N=128 \\
\text { gender }= \\
\text { NR }\end{array}$ & $\begin{array}{l}\mathrm{G} 1: N=57, \text { range } 10^{\circ}-20^{\circ}, \\
13.7^{\circ} \pm 2.6 \\
\mathrm{G} 2: N=34, \text { range } 20^{\circ}-30^{\circ}, \\
23.9^{\circ} \pm 3.2 \\
\mathrm{G} 3: N=37, \text { range }>40^{\circ}, 36.4^{\circ} \\
\pm 5.8\end{array}$ & $\begin{array}{l}\mathrm{G} 1: 15.5 \\
\pm 1.8 \\
\mathrm{G} 2: 15.1 \\
\pm 1.6 \\
\mathrm{G} 3: 15.5 \\
\pm 1.9\end{array}$ & $\begin{array}{l}\text { G1: } 160 \pm 6,51.2 \pm \\
9.1 \\
\text { G2: } 160 \pm 6,47.3 \pm \\
8.1 \\
\text { G3: } 160 \pm 7,50.0 \pm \\
8.5\end{array}$ \\
\hline $\begin{array}{l}\text { de } \\
\text { Santiago } \\
\text { et al. } 2013 \\
\text { [44] }\end{array}$ & $\begin{array}{l}\text { CON: NR } \\
\text { AIS: Hospital, Brazil }\end{array}$ & $\begin{array}{l}N=15 \\
\text { gender }= \\
15: 0\end{array}$ & $\begin{array}{l}15.1 \\
\pm 1.5\end{array}$ & $\begin{array}{l}159.7 \pm 0.0 \\
51.2 \pm 2.0\end{array}$ & $\begin{array}{l}N=15 \\
\text { gender }= \\
15: 0\end{array}$ & $69.5^{\circ} \pm 8.78$, right thoracic & $\begin{array}{l}15.0 \pm \\
1.6\end{array}$ & $\begin{array}{l}156.8 \pm 0.03,46.1 \pm \\
3.3\end{array}$ \\
\hline $\begin{array}{l}\text { Stylianides } \\
\text { et al. } 2013 \\
{[45]}\end{array}$ & $\begin{array}{l}\text { CON: NR } \\
\text { AIS: Hospital, } \\
\text { Canada }\end{array}$ & $\begin{array}{l}N=25 \\
\text { gender }= \\
25: 0\end{array}$ & $\begin{array}{l}13.1 \\
\pm 1.4\end{array}$ & $\begin{array}{l}156.9 \pm 6.9 \\
46.0 \pm 7.4\end{array}$ & $\begin{array}{l}N=28 \\
\text { Gender }= \\
28: 0\end{array}$ & $35.0^{\circ} \pm 7.6$, right thoracic & $\begin{array}{l}12.9 \pm \\
1.6\end{array}$ & $155.1 \pm 9.9,44.7 \pm 9.5$ \\
\hline $\begin{array}{l}\text { Gruber et } \\
\text { al. } 2011 \\
{[46]}\end{array}$ & $\begin{array}{l}\text { CON: NR } \\
\text { AIS: Hospital, } \\
\text { Mexico }\end{array}$ & $\begin{array}{l}N=10 \\
\text { gender }= \\
10: 0\end{array}$ & $\begin{array}{l}11.9 \\
\pm 2.8\end{array}$ & $\begin{array}{l}149.0 \pm 14.0 \\
44.5 \pm 7.7\end{array}$ & $\begin{array}{l}N=36 \\
\text { gender }= \\
36: 0\end{array}$ & $\begin{array}{l}\mathrm{G} 1: N=18 \text { pre-brace } 27^{\circ} \pm 6 \\
\mathrm{G} 2: N=18 \text { pre-op } 52^{\circ} \pm 13 \\
\text { Right thoracic predominantly }\end{array}$ & $\begin{array}{l}12.5 \pm \\
2.0\end{array}$ & $\begin{array}{l}154 \pm 11.0,48.6 \pm \\
12.5\end{array}$ \\
\hline $\begin{array}{l}\text { Dalleau et } \\
\text { al. } 2011 \\
{[47]}\end{array}$ & $\begin{array}{l}\text { CON: NR } \\
\text { AIS: NR }\end{array}$ & $\begin{array}{l}N=20 \\
\text { gender }= \\
20: 0\end{array}$ & $\begin{array}{l}12.5 \\
\pm 1.3\end{array}$ & $\begin{array}{l}156.3 \pm 7.7 \\
43.7 \pm 6.9\end{array}$ & $\begin{array}{l}N=21 \\
\text { gender }= \\
21: 0\end{array}$ & $\begin{array}{l}\text { range } 5^{\circ}-28^{\circ} \\
13.5^{\circ} \pm 5.5, \text { Right thoracic }\end{array}$ & $\begin{array}{l}11.7 \pm \\
3.1\end{array}$ & $\begin{array}{l}148.4 \pm 17.0,40.0 \pm \\
13.3\end{array}$ \\
\hline $\begin{array}{l}\text { Valles et al. } \\
2009 \text { [48] }\end{array}$ & $\begin{array}{l}\text { CON: NR } \\
\text { AIS: NR }\end{array}$ & $\begin{array}{l}N=20 \\
\text { gender }= \\
\text { NR }\end{array}$ & NR & $N R, N R$ & $\begin{array}{l}N=16 \\
\text { gender }= \\
13: 3\end{array}$ & NR & $\begin{array}{l}14.8 \pm \\
2.1\end{array}$ & $\begin{array}{l}151.9 \pm 30.7,59.8 \pm \\
14.4\end{array}$ \\
\hline $\begin{array}{l}\text { Beaulieu et } \\
\text { al. } 2009 \\
\text { [49] }\end{array}$ & $\begin{array}{l}\text { CON: Community, } \\
\text { Canada } \\
\text { AIS: Hospital, } \\
\text { Canada }\end{array}$ & $\begin{array}{l}N=53 \\
\text { gender }= \\
53: 0\end{array}$ & $\begin{array}{l}13.8 \\
\pm 1.0\end{array}$ & $\begin{array}{l}159.2 \pm 9.3 \\
49.9 \pm 9.8\end{array}$ & $\begin{array}{l}N=49 \\
\text { gender }= \\
\text { NR }\end{array}$ & $\begin{array}{l}\mathrm{G} 1: \mathrm{N}=23 \mathrm{OB}, 18.9^{\circ} \pm 7.1, \mathrm{~N}= \\
20 \text { Right thoracic, } N=3 \text { Right } \\
\text { lumbar } \\
\mathrm{G} 2: \mathrm{N}=26 \mathrm{~PB}, 27.2^{\circ} \pm 12.4, \mathrm{~N} \\
=25 \text { Right thoracic, } \mathrm{N}=1 \\
\text { Right lumbar }\end{array}$ & $\begin{array}{l}\text { G1: } \\
12.5 \pm \\
2.4 \\
\text { G2: } \\
12.2 \pm \\
1.4\end{array}$ & $\begin{array}{l}\text { G1: } 151.5 \pm 10.7 \\
43.5 \pm 10.9, G 2: \\
152.3 \pm 10.3,42.1 \pm \\
8.3\end{array}$ \\
\hline $\begin{array}{l}\text { Zabjek et } \\
\text { al. 2008 } \\
\text { [50] }\end{array}$ & $\begin{array}{l}\text { Canada, CON: NR, } \\
\text { AIS: NR }\end{array}$ & $\begin{array}{l}N=18 \\
\text { gender }= \\
18: 0\end{array}$ & $\begin{array}{l}11.0 \\
\pm 2.0\end{array}$ & $\begin{array}{l}144.0 \pm 13.0 \\
39.0 \pm 11.0\end{array}$ & $\begin{array}{l}N=22 \\
\text { gender }= \\
22: 0\end{array}$ & $\begin{array}{l}21.0^{\circ} \pm 14.0, N=8 \text { Double, } N= \\
7 \text { Thoracolumbar, } N=7 \\
\text { Thoracic }\end{array}$ & $\begin{array}{l}12.2 \pm \\
2.0\end{array}$ & $\begin{array}{l}148.0 \pm 11.0,42.0 \pm \\
12.0\end{array}$ \\
\hline $\begin{array}{l}\text { Dalleau et } \\
\text { al. } 2007 \\
{[51]}\end{array}$ & $\begin{array}{l}\text { CON: NR } \\
\text { AIS: NR }\end{array}$ & $\begin{array}{l}N=23 \\
\text { gender }= \\
23: 0\end{array}$ & $\begin{array}{l}13.4 \\
\pm 1.0\end{array}$ & $\begin{array}{l}161.5 \pm 5.9 \\
50.0 \pm 11.1\end{array}$ & $\begin{array}{l}N=23 \\
\text { gender }= \\
23: 0\end{array}$ & $29.4^{\circ} \pm 9.4$, right thoracic & $\begin{array}{l}12.2 \pm \\
1.5\end{array}$ & $\begin{array}{l}154.0 \pm 10.5,44.4 \pm \\
9.8\end{array}$ \\
\hline $\begin{array}{l}\text { Simoneau } \\
\text { et al. } 2006 \\
\text { [52] }\end{array}$ & $\begin{array}{l}\text { Canada, CON: NR, } \\
\text { AIS: NR }\end{array}$ & $\begin{array}{l}N=9, \\
\text { gender }= \\
9: 0\end{array}$ & $\begin{array}{l}16.5 \\
\pm 1.7\end{array}$ & $N R, N R$ & $\begin{array}{l}N=8 \\
\text { gender }= \\
7: 1\end{array}$ & $45.6^{\circ} \pm 7.5$, right thoracic & $\begin{array}{l}16.3 \pm \\
2.1\end{array}$ & $N R, N R$ \\
\hline $\begin{array}{l}\text { Chow et } \\
\text { al. } 2006 \\
\text { [53] }\end{array}$ & $\begin{array}{l}\text { Canada, CON: NR, } \\
\text { AIS: NR }\end{array}$ & $\begin{array}{l}N=20 \\
\text { gender }= \\
20: 0\end{array}$ & $\begin{array}{l}13.5 \\
\pm 1.1\end{array}$ & $\begin{array}{l}155 \pm 6,44.8 \\
\pm 5.1\end{array}$ & $\begin{array}{l}N=26 \\
\text { gender }= \\
26: 0\end{array}$ & $\begin{array}{l}21.0^{\circ} \pm 3.0, N=10 \text { right } \\
\text { primary, } N=16 \text { left primary }\end{array}$ & $\begin{array}{l}13.0 \pm \\
0.9\end{array}$ & $156.0 \pm 5.0,43.9 \pm 5.8$ \\
\hline $\begin{array}{l}\text { Simoneau } \\
\text { et al. } 2006 \\
\text { [54] }\end{array}$ & $\begin{array}{l}\text { Canada, CON: NR, } \\
\text { AIS: NR }\end{array}$ & $\begin{array}{l}N=10 \\
\text { gender }= \\
10: 0\end{array}$ & 16.5 & $N R, N R$ & $\begin{array}{l}N=8 \\
\text { gender }= \\
7: 1\end{array}$ & $45.6^{\circ} \pm 7.5$ & 16.4 & NR \\
\hline $\begin{array}{l}\text { Allard et al. } \\
2004 \text { [55] }\end{array}$ & $\begin{array}{l}\text { CON: Community } \\
\text { AIS: NR }\end{array}$ & $\begin{array}{l}N=36 \\
\text { gender }= \\
36: 0\end{array}$ & $\begin{array}{l}13.5 \\
\pm 1.7\end{array}$ & $\begin{array}{l}159.2 \pm 8.4 \\
49.1 \pm 10.3\end{array}$ & $\begin{array}{l}N=38 \\
\text { gender }= \\
38: 0\end{array}$ & $26.2^{\circ} \pm 11.5$, right thoracic & $\begin{array}{l}12.4 \pm \\
1.8\end{array}$ & $\begin{array}{l}151.4 \pm 11.2,42.2 \pm \\
9.3\end{array}$ \\
\hline $\begin{array}{l}\text { Silferi et al. } \\
2004 \text { [56] }\end{array}$ & $\begin{array}{l}\text { CON: NR } \\
\text { AIS:NR }\end{array}$ & $\begin{array}{l}N=15 \\
\text { gender }= \\
15: 0\end{array}$ & $\begin{array}{l}\text { range } \\
11-16\end{array}$ & $N R, N R$ & $\begin{array}{l}N=15 \\
\text { gender }= \\
15: 0\end{array}$ & NR & $\begin{array}{l}\text { range } \\
11-16\end{array}$ & $N R, N R$ \\
\hline $\begin{array}{l}\text { Nault et al. } \\
2002\end{array}$ & $\begin{array}{l}\text { Canada, CON: NR, } \\
\text { AIS: NR }\end{array}$ & $\begin{array}{l}N=38 \\
\text { gender }=\end{array}$ & $\begin{array}{l}12.9 \\
\pm 2.0\end{array}$ & $\begin{array}{l}156.7 \pm 10.8 \\
45.3 \pm 8.5\end{array}$ & $\begin{array}{l}N=43 \\
\text { gender }=\end{array}$ & $\begin{array}{l}29^{\circ} \pm 12, \text { range } 7^{\circ}-52^{\circ}, N=39 \\
\text { right thoracic, } N=2\end{array}$ & $\begin{array}{l}12.5 \pm \\
1.7\end{array}$ & $153.1 \pm 9.7,43.2 \pm 9.1$ \\
\hline
\end{tabular}


Table 6 Participant characteristics of CON and AIS from all included studies (Continued)

\begin{tabular}{|c|c|c|c|c|c|c|c|c|}
\hline \multirow{2}{*}{$\begin{array}{l}\text { Author } \\
\text { Year }\end{array}$} & \multirow{2}{*}{$\begin{array}{l}\text { Country and } \\
\text { setting of participant } \\
\text { recruitment }\end{array}$} & \multicolumn{3}{|l|}{ CON } & \multicolumn{4}{|l|}{ AIS } \\
\hline & & $\begin{array}{l}N=\text { Sample } \\
\text { size, gender } \\
=\text { o }:{ }^{N}\end{array}$ & $\begin{array}{l}\text { Age } \\
\pm \mathrm{SD} \\
\text { (years) }\end{array}$ & $\begin{array}{l}\text { Height } \pm \text { SD } \\
(\mathrm{cm}) \text {, weight } \\
\pm \mathrm{SD}(\mathrm{kg})\end{array}$ & $\begin{array}{l}N=\text { Sample } \\
\text { size, } \\
\text { gender } \\
=+: 0^{\lambda}\end{array}$ & $\begin{array}{l}\text { Cobb }\left(^{\circ}\right) \text { : range and/or mean } \\
\pm \mathrm{SD} \text {, primary curve type }\end{array}$ & $\begin{array}{l}\text { Age } \pm \\
\text { SD } \\
\text { (years) }\end{array}$ & $\begin{array}{l}\text { Height } \pm \text { SD }(\mathrm{cm}), \\
\text { weight } \pm S D(\mathrm{~kg})\end{array}$ \\
\hline [57] & & $38: 0$ & & & 43:0 & thoracolumbar, $N=2$ lumbar & & \\
\hline $\begin{array}{l}\text { Chen et al. } \\
1998 \\
{[58]}\end{array}$ & $\begin{array}{l}\text { CON: NR } \\
\text { AIS: Hospital, } \\
\text { Taiwan }\end{array}$ & $\begin{array}{l}N=15 \\
\text { gender }= \\
13: 2\end{array}$ & $\begin{array}{l}16.8 \\
\pm 3.1\end{array}$ & $N R, N R$ & $\begin{array}{l}N=30 \\
\text { gender }= \\
28: 2\end{array}$ & range $22^{\circ}-67^{\circ}$ & $\begin{array}{l}16.6 \pm \\
3.8\end{array}$ & $N R, N R$ \\
\hline $\begin{array}{l}\text { Sahlstrand } \\
\text { et al. } 1978 \\
\text { [59] }\end{array}$ & $\begin{array}{l}\text { CON: NR } \\
\text { AIS: Hospital, } \\
\text { Sweden }\end{array}$ & $\begin{array}{l}N=48 \\
\text { gender }= \\
40: 8\end{array}$ & $\begin{array}{l}13.4 \\
\pm 1.7\end{array}$ & $N R, N R$ & $\begin{array}{l}N=32 \\
\text { gender }= \\
27: 5\end{array}$ & NR & $\begin{array}{l}13.4 \pm \\
2.1\end{array}$ & NR \\
\hline
\end{tabular}

$G$ group, $N$ number, $N R$ not reported, $O B$ observation group, $P B$ pre-bracing group, $S D$ standard deviation

mean Cobb angles, of which 4 studies [47, 49, 50, 53] participants were classified as mild with a distribution from $5^{\circ}$ to $28^{\circ}, 6$ studies $[43,45,46,51,55,57]$ as moderate $\left(25^{\circ}-45^{\circ}\right), 2[52,54]$ as severe $\left(>45^{\circ}\right)$ and 1 as high severity $\left(>60^{\circ}\right)$ [44]. Two studies reported Cobb angle ranges [42, 58], and for three studies, Cobb angles were not reported $[48,56,59]$. A total of 12 studies [42, 44-47, 49-53, 55, 57] reported a primary location or side of curvature, and right thoracic primary curves were predominant in 9 studies [44$47,49,51,52,55,57]$.

\section{Posturography method used to measure postural stability} The description of posturography method (Table 7) displays type of force plate, study protocol, COP parameters and values in each study. In 13 studies [45-57], the AMTI force plate (AMTI, Newton, MA, USA) was the most common to assess postural stability. A standard COP parameter sampling frequency ranged from 20 to $1080 \mathrm{~Hz}$ with $64 \mathrm{~Hz}$ cited in 8 studies [45, 47, 49, 51, 53, 55-57]. Two studies used $20 \mathrm{~Hz}$ [50] and $100 \mathrm{~Hz}$ [48] with two independent force plates under each foot. A variation in study protocols was observed with spacing of participant's feet ranging from heels placed together [59] or shoulder width apart $[44,46]$. Eleven studies specified the degree in which participant's feet were externally rotated with $15^{\circ}$ noted in 7 studies [45, 47, 49, 51, 55-57]. Disparity in trial durations and repetitions among the literature was evident. Trial duration varied from $10 \mathrm{~s}$ to $2 \mathrm{~min}$, with $64 \mathrm{~s}$ being the most common $[45,49,51,55,56]$. The number of trials ranged from 1 to 6 trials. Three out of the 18 studies used study protocols which involved participants barefoot with heels spaced $23 \mathrm{~cm}$ apart, feet pointing externally $15^{\circ}$, vision focused on a target placed $1.2 \mathrm{~m}$ in front at eye level and three trials of $64 \mathrm{~s}[45,51,55]$.

\section{Qualitative analysis of centre of pressure parameters}

Description of data extracted from all included articles and findings of COP parameters and available statistical significance between groups are summarised in Table 7 .

\section{Sway area}

Of 18 included studies, 9 investigated sway area [42, 44, $46,48,49,55,57-59]$ (Table 7). Seven studies reported significantly higher mean sway area values in AIS compared to CON [42, 44, 46, 55, 57-59]. One study [49] divided AIS subjects into pre-bracing (PB) and observation groups (OB). The PB group displayed 58\% higher values than the $\mathrm{CON}$, which was significant $(p=$ 0.008). The $\mathrm{OB}$ showed $15 \%$ higher values than the $\mathrm{CON}$; however, this was not a significant difference (NS).

\section{Mediolateral}

Of 18 included studies, 13 reported COP in ML position and range [42, 45-47, 49-51, 54-59] (Table 7). Two studies reported ML measurements as both position and range $[49,51]$. Six studies reported position located towards the right $[49-51,55,57,59]$; however, only one study [59] reported significant $(p<0.05)$ difference between AIS and CON. Eight studies reported higher values in the ML range for the AIS group compared to the $\mathrm{CON}$ with 7 studies reporting significant differences [45-47, 49, 51, 58, 59] and 6 noticed ML positional shift towards right $[49-51,55,57,59]$.

\section{Anteroposterior}

Of 18 included studies, 14 reported COP in AP position and range [42, 45-47, 49-51, 53-59] (Table 7). Two studies reported AP measurements both as position and range $[49,51]$. Eight studies reported higher values in the AP range for the AIS group compared to the CON with 5 studies reporting significant differences [45, 47, $49,51,59]$. The direction of AP position was significantly $(p<0.05)$ located posteriorly towards heels for AIS compared to CON in 5 of the included studies [42, $49,51,55,57]$.

\section{Meta-analysis of COP parameters}

To ensure consistency across the quantitative meta-analysis, a further 9 studies were removed due to 
Table 7 Description of data extracted from all included articles

\begin{tabular}{|c|c|c|c|c|}
\hline $\begin{array}{l}\text { Author } \\
\text { Year }\end{array}$ & Force platform & Study protocol & $\begin{array}{l}\text { COP parameters and direction } \\
\text { of displacement }\end{array}$ & Values for AIS, CON, $p$ value \\
\hline \multirow[t]{3}{*}{$\begin{array}{l}\text { Sahli et al. } \\
2013 \text { [42] }\end{array}$} & \multirow{3}{*}{$\begin{array}{l}\text { Static Stabilometric } \\
\text { platform (SATEL, } \\
\text { Blagnac, France), } 40 \mathrm{~Hz}\end{array}$} & \multirow{3}{*}{$\begin{array}{l}\text { Heels } 5 \mathrm{~cm} \text { apart, } 30^{\circ} \text { angle between } \\
\text { long axes of the feet. Eye focused } \\
\text { at eye level, white cross on wall } 2 \\
\mathrm{~m} \text { away, } 3 \text { trials of } 51.2 \mathrm{~s}, 30-\mathrm{s} \\
\text { rest interval }\end{array}$} & Mean \pm SD Position of ML & $\begin{array}{l}\text { \#AIS }=22 \pm 13.1 \mathrm{~mm}, \# C O N=17 \pm \\
26 \mathrm{~mm}, p<0001^{*}\end{array}$ \\
\hline & & & Mean \pm SD Positon of AP & $\begin{array}{l}\# A I S=25 \pm 37.4 \mathrm{~mm}, \# C O N=22 \pm \\
22.5 \mathrm{~mm}, p<0.001^{*}\end{array}$ \\
\hline & & & Mean \pm SD Sway Area & $\begin{array}{l}\# \text { AIS }=490 \pm 318 \mathrm{~mm}^{2}, \# C O N=310 \pm \\
364 \mathrm{~mm}^{2}, p<0.001^{*}\end{array}$ \\
\hline \multirow[t]{2}{*}{$\begin{array}{l}\text { Park et al. } \\
2013 \text { [43] }\end{array}$} & \multirow[t]{2}{*}{$\begin{array}{l}\text { MFT balance tester } \\
\text { (MFT balance test- } \\
\text { basic, Multifunktionale } \\
\text { trainsgerate, Germany) }\end{array}$} & \multirow[t]{2}{*}{$\begin{array}{l}\text { Barefoot, subjects were to locate } \\
\text { their centre of gravity into } 5 \\
\text { different sections from the } \\
\text { centre of a circle, } 2 \text { trials of } 30 \mathrm{~s}\end{array}$} & $\begin{array}{l}\times \text { Mean left and right balance } \\
\text { in } \mathrm{kg} / \mathrm{m}^{2} \text { derived from the } \\
\text { absolute values by subtracting } \\
\text { the rate of the opposite } \\
\text { direction from the } \\
\text { rate of the selected direction }\end{array}$ & $\begin{array}{l}\text { AIS G1 }=12.57 \pm 9.55 \mathrm{~kg} / \mathrm{m}^{2}, \mathrm{G} 2=13.47 \\
\pm 11.54 \mathrm{~kg} / \mathrm{m}^{2} \\
\mathrm{G} 3=12.33 \pm 10.68 \mathrm{~kg} / \mathrm{m}^{2}, \mathrm{CON}=2.38 \pm \\
1.96 \mathrm{~kg} / \mathrm{m}^{2} \\
p<0.01 \text { between all groups }\end{array}$ \\
\hline & & & $\begin{array}{l}\text { ×Mean forward and backward } \\
\text { balance in } \mathrm{kg} / \mathrm{m}^{2} \text { derived } \\
\text { from the absolute values by } \\
\text { subtracting the rate of the } \\
\text { opposite direction from } \\
\text { the rate of the } \\
\text { selected direction }\end{array}$ & $\begin{array}{l}\text { AIS G1 }=20.44 \pm 12.91 \mathrm{~kg} / \mathrm{m}^{2}, \mathrm{G} 2= \\
22.14 \pm 18.03 \mathrm{~kg} / \mathrm{m}^{2}, \mathrm{G} 3=16.28 \pm \\
11.43 \mathrm{~kg} / \mathrm{m}^{2}, \mathrm{CON}=10.37 \pm 8.51 \mathrm{~kg} / \mathrm{m}^{2}, \\
p<0.01 \text { between all groups }\end{array}$ \\
\hline $\begin{array}{l}\text { de } \\
\text { Santiago } \\
\text { et al. } 2013 \\
\text { [44] }\end{array}$ & $\begin{array}{l}\text { EMG system force } \\
\text { platform } \\
\text { (Sao Jose dos Campos, } \\
\text { Brazil), } 100 \mathrm{~Hz}\end{array}$ & $\begin{array}{l}\text { Feet shoulder width apart, arms } \\
\text { along body, eyes focused } \\
\text { at eye level at } 5 \mathrm{~cm} \\
\text { diameter black circle } 1.5 \mathrm{~m} \\
\text { away, } 3 \text { trials of } 60 \mathrm{~s} \text {, self-chosen rest }\end{array}$ & $x$ Mean \pm SD Sway Area & $\begin{array}{l}\# A I S=60 \pm 1.4 \mathrm{~mm}^{2}, \# C O N=40 \pm \\
2.8 \mathrm{~mm}^{2}, p<0.0001^{*}\end{array}$ \\
\hline \multirow{2}{*}{$\begin{array}{l}\text { Stylianides } \\
\text { et al. } 2013 \\
{[45]}\end{array}$} & \multirow{2}{*}{$\begin{array}{l}\text { AMTI force platform } \\
\text { model OR6-5 (Newton, } \\
\text { MA), } 64 \mathrm{~Hz}\end{array}$} & \multirow{2}{*}{$\begin{array}{l}\text { Barefoot, heels } 23 \mathrm{~cm} \text { apart, } \\
\text { feet external rotated } \\
15^{\circ} \text {, eyes focused at eye } \\
\text { level target } 1.2 \mathrm{~m} \text { away, } \\
3 \text { trials of } 64 \mathrm{~s}\end{array}$} & Mean \pm SD Range of ML & $\begin{array}{l}\text { \#AIS }=19.8 \pm 9 \mathrm{~mm}, \# C O N=14.5 \pm \\
6.3 \mathrm{~mm}, p<0.05^{*}\end{array}$ \\
\hline & & & $\begin{array}{l}\text { Mean } \pm \text { SD Range of AP, } \\
\text { posterior displacement }\end{array}$ & $\begin{array}{l}\# A I S=32 \pm 13.3 \mathrm{~mm}, \# C O N=25.8 \pm \\
7.8 \mathrm{~mm}, p<0.05^{*}\end{array}$ \\
\hline \multirow{3}{*}{$\begin{array}{l}\text { Gruber et } \\
\text { al. } 2011 \\
{[46]}\end{array}$} & \multirow{3}{*}{$\begin{array}{l}\text { AMTI force platform } \\
\text { (AMTI, Newton, MA), } \\
1080 \mathrm{~Hz}\end{array}$} & \multirow{3}{*}{$\begin{array}{l}\text { Feet shoulder width apart, } \\
\text { hands by the side, looking } \\
\text { straight ahead, } 3 \text { trials of } 10 \mathrm{~s}\end{array}$} & Mean \pm SD Range of ML & $\begin{array}{l}\text { AlS }=28.99 \pm 25.55 \mathrm{~mm}, \mathrm{CON}=17.25 \pm \\
7.09 \mathrm{~mm}, p=0.025^{*}\end{array}$ \\
\hline & & & Mean \pm SD Range of AP & $\begin{array}{l}\mathrm{AlS}=28.39 \pm 11.44 \mathrm{~mm}, \mathrm{CON}=25.00 \pm \\
11.72 \mathrm{~mm}, p=\mathrm{NS}\end{array}$ \\
\hline & & & Mean \pm SD Sway Area & $\begin{array}{l}\mathrm{AIS}=3.73 \pm 0.40 \mathrm{~mm}^{2}, \mathrm{CON}=3.48 \pm \\
0.38 \mathrm{~mm}^{2}, p<0.005^{*}\end{array}$ \\
\hline \multirow{2}{*}{$\begin{array}{l}\text { Dalleau et } \\
\text { al. } 2011 \\
{[47]}\end{array}$} & \multirow{2}{*}{$\begin{array}{l}\text { AMTI force platform } \\
\text { model OR-5 (Newton, } \\
\text { MA, USA), } 64 \mathrm{~Hz}\end{array}$} & \multirow{2}{*}{$\begin{array}{l}\text { Barefoot, heels } 23 \mathrm{~cm} \text { apart, } \\
\text { feet pointing externally } \\
15^{\circ} \text {, eyes focused on target } \\
1.2 \mathrm{~m} \text { ahead, } 3 \text { trials of } 30 \mathrm{~s}\end{array}$} & ×Median (IQR) Range of ML & $\begin{array}{l}\text { \#AIS }=15.0 \mathrm{~mm}(6.5 \mathrm{~mm}), \# \mathrm{CON}= \\
12.0 \mathrm{~mm}(7.0 \mathrm{~mm}), p=0.02^{*}\end{array}$ \\
\hline & & & $\begin{array}{l}\text { ×Median (IQR) Range of AP, } \\
\text { posterior displacement }\end{array}$ & $\begin{array}{l}\text { \#AIS }=25.5 \mathrm{~mm}(8.2 \mathrm{~mm}), \# C O N= \\
16.5 \mathrm{~mm}(7.2 \mathrm{~mm}), p<0.01^{*}\end{array}$ \\
\hline $\begin{array}{l}\text { Valles et al. } \\
2009[48]\end{array}$ & $\begin{array}{l}\text { 2xAMTI force plates } \\
\text { under each foot model } \\
\text { 0TS6-500, } 100 \mathrm{~Hz}\end{array}$ & 3 trials of $30 \mathrm{~s}$ & $\times$ Mean \pm SD Sway Area & $\begin{array}{l}\mathrm{AIS}=2728 \pm 4177 \mathrm{~mm}^{2}, \mathrm{CON}=2152 \pm \\
2767 \mathrm{~mm}^{2}, p=\mathrm{NS}\end{array}$ \\
\hline \multirow[t]{3}{*}{$\begin{array}{l}\text { Beaulieu et } \\
\text { al. } 2009 \\
{[49]}\end{array}$} & \multirow[t]{3}{*}{$\begin{array}{l}\text { AMTI force platform } \\
\text { (Newton, MA, USA), } \\
64 \mathrm{~Hz}\end{array}$} & \multirow[t]{3}{*}{$\begin{array}{l}\text { Heels spaced } 20 \mathrm{~cm} \text { apart, } \\
\text { feet pointing externally } \\
15^{\circ} \text {, eyes focused on } \\
\text { target } 1.2 \mathrm{~m} \text { away, } 3 \text { trials of } 64 \mathrm{~s} \text {, } \\
\text { 2-min rest intervals }\end{array}$} & $\begin{array}{l}\text { Mean } \pm \text { SD Position of } \mathrm{ML} \text {, } \\
\text { right displacement } \\
\text { Mean } \pm \text { SD Range of } M L\end{array}$ & $\begin{array}{l}\text { \#AIS }=4.58 \pm 11.5 \mathrm{~mm}(\mathrm{OB} \& \mathrm{~PB}), \# C O N \\
=4.0 \pm 9.0 \mathrm{~mm}, p=\mathrm{NS} \text { between OB, PB } \\
\text { and CON groups. } \\
\text { \#AIS }=541.3 \pm 244.9 \mathrm{~mm}, \text { \#CON }=184.6 \\
\pm 153.8 \mathrm{~mm}, p<0.001^{*} \mathrm{OB} \text { vs CON, } p< \\
0.001^{*} \mathrm{~PB} \text { vs CON }\end{array}$ \\
\hline & & & $\begin{array}{l}\text { Mean } \pm \text { SD Position of AP, } \\
\text { posterior displacement } \\
\text { Mean } \pm \text { SD Range of AP }\end{array}$ & $\begin{array}{l}\# A I S=73.8 \pm 13.0 \mathrm{~mm}(\mathrm{OB} \& \mathrm{~PB}), \# C O N \\
=64.0 \pm 13.0 \mathrm{~mm}, p=0.04^{*} \mathrm{OB} \text { vs CON, } \\
p=0.001^{*} \mathrm{~PB} \text { vs CON } \\
\text { \#AIS }=629.0 \pm 156.7 \mathrm{~mm}, \# \mathrm{CON}=233.3 \\
\pm 186.2 \mathrm{~mm}, \mathrm{p}<0.001^{*} \mathrm{OB} \text { vs CON, } p< \\
0.001^{*} \mathrm{~PB} \text { vs CON }\end{array}$ \\
\hline & & & Mean \pm SD Sway Area & 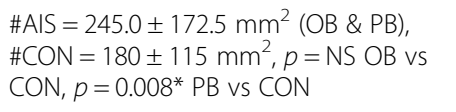 \\
\hline Zabjek et & $2 \times$ AMTI force & Quiet standing position, & xMean \pm SD Position & AIS $=3.7 \pm 11,08 \mathrm{~mm}, \mathrm{CON}=4.6 \pm$ \\
\hline
\end{tabular}


Table 7 Description of data extracted from all included articles (Continued)

\begin{tabular}{|c|c|c|c|c|}
\hline $\begin{array}{l}\text { Author } \\
\text { Year }\end{array}$ & Force platform & Study protocol & $\begin{array}{l}\text { COP parameters and direction } \\
\text { of displacement }\end{array}$ & Values for AIS, CON, $p$ value \\
\hline \multirow[t]{2}{*}{$\begin{array}{l}\text { al. } 2008 \\
\text { [50] }\end{array}$} & \multirow[t]{2}{*}{$\begin{array}{l}\text { platforms under each } \\
\text { foot, } 20 \mathrm{~Hz}\end{array}$} & \multirow[t]{2}{*}{$\begin{array}{l}4 \text { trials of } 2 \text { min, adequate } \\
\text { rest between each trial }\end{array}$} & $\begin{array}{l}\text { estimate gravity line } \\
\text { of COM ML, right } \\
\text { displacement }\end{array}$ & $9,69 \mathrm{~mm}, p=\mathrm{NR}$ \\
\hline & & & $\begin{array}{l}\times \text { Mean } \pm \text { SD Position } \\
\text { estimate gravity line } \\
\text { of COM in AP, anterior } \\
\text { displacement }\end{array}$ & $\begin{array}{l}\mathrm{AIS}=30.2 \pm 16,06 \mathrm{~mm}, \mathrm{CON}=28.3 \pm \\
16,16 \mathrm{~mm}, p=\mathrm{NR}\end{array}$ \\
\hline \multirow[t]{2}{*}{$\begin{array}{l}\text { Dalleau et } \\
\text { al. } 2007 \\
{[51]}\end{array}$} & \multirow[t]{2}{*}{$\begin{array}{l}\text { AMTI force platform } \\
\text { (Newton, MA, USA), } \\
64 \mathrm{~Hz}\end{array}$} & \multirow{2}{*}{$\begin{array}{l}\text { Barefoot, heels spaced } 23 \mathrm{~cm} \\
\text { apart, feet pointing externally } \\
15^{\circ} \text {, arms along body, } \\
\text { eyes focused at eye level } \\
\text { target } 1.2 \mathrm{~m} \text { away, } \\
3 \text { trials of } 64 \mathrm{~s}, 60 \text {-s rest intervals }\end{array}$} & $\begin{array}{l}\text { Mean } \pm \text { SD Position of } M L, \\
\text { right displacement } \\
\text { Mean } \pm \text { SD Range of ML }\end{array}$ & $\begin{array}{l}\mathrm{AIS}=3.3 \pm 12.0 \mathrm{~mm}, \mathrm{CON}=3.4 \pm \\
6.8 \mathrm{~mm}, p=\mathrm{NS} \\
\# \mathrm{AIS}=18 \pm 3.5 \mathrm{~mm}, \# \mathrm{CON}=13 \pm \\
2.5 \mathrm{~mm}, p=0.001^{*}\end{array}$ \\
\hline & & & $\begin{array}{l}\text { Mean } \pm \text { SD Position of AP, } \\
\text { posterior displacement } \\
\text { Mean } \pm \text { SD Range of AP }\end{array}$ & $\begin{array}{l}\mathrm{AIS}=62.3 \pm 10.7 \mathrm{~mm}, \mathrm{CON}=71.3 \pm \\
14.3 \mathrm{~mm}, p=0.043^{*} \\
\# \mathrm{AIS}=29.5 \pm 8 \mathrm{~mm}, \# \mathrm{CON}=24 \pm 7 \mathrm{~mm}, \\
p=0.016^{*}\end{array}$ \\
\hline $\begin{array}{l}\text { Simoneau } \\
\text { et al. } \\
\text { 2006a [52] }\end{array}$ & $\begin{array}{l}\text { AMTI force platform } \\
\text { model OR6-6 } \\
\text { (Watertown, USA), }\end{array}$ & $\begin{array}{l}\text { Barefoot with feet } 10 \mathrm{~cm} \\
\text { apart, arms along body, } \\
\text { eyes focused at eye level }\end{array}$ & $\begin{array}{l}\times \text { Mean distance between } \\
\text { consecutive zones of } 3 \mathrm{~mm} \\
\text { radius, sway density curve }\end{array}$ & $\begin{array}{l}\# A I S=3.8 \mathrm{~mm} \pm 2,3, \# C O N=1.69 \mathrm{~mm} \pm \\
2,14, p<0.01^{*}\end{array}$ \\
\hline
\end{tabular}

2006a [52] (Watertown, USA), $200 \mathrm{~Hz}$

Chow et AMTI force platform al. 2006 (Newton, MA, USA), [53] $\quad 64 \mathrm{~Hz}$

Simoneau AMTI force platform,

et al. $200 \mathrm{~Hz}$

2006b [54]

Allard et al. AMTI force platform 2004 [55] (Newton, MA, USA) $64 \mathrm{~Hz}$ eyes focused at eye leve target $2 \mathrm{~m}$ away, 6 trials of $30 \mathrm{~s}$ divided into two 15-s intervals

Barefoot, heels $10 \mathrm{~cm}$ apart, feet pointing externally $30^{\circ}$, arms along body Eyes focused at eye level $10 \mathrm{~cm} \times 15 \mathrm{~cm}$ reference square $2 \mathrm{~m}$ away, 3 trials of $60 \mathrm{~s}, 3$-min rest intervals

Barefoot with feet $10 \mathrm{~cm}$ apart, arms along body, eyes focused at eye level target $2 \mathrm{~m}$ away, 6 trials of $15 \mathrm{~s}$

Heels spaced $23 \mathrm{~cm}$ apart, feet pointing externally

$15^{\circ}$, eyes focused on target $1.2 \mathrm{~m}$ away, 3 trials of $64 \mathrm{~s}$

Silferi et al. AMTI force platform 2004 [56] (Newton, MA), $64 \mathrm{~Hz}$

Nault et al. AMTI force platform 2002 [57] (Newton, MA) at $64 \mathrm{~Hz}$

Chen et al. Kistler Instrument Corp 1998 [58] at $50 \mathrm{~Hz}$
Heels spaced $20 \mathrm{~cm}$ apart, feet external rotation $15^{\circ}$. Focused ahead at a target $1.2 \mathrm{~m}$ away, 3 trials of $64 \mathrm{~s}$, 30-s rests

Heels spaced $20 \mathrm{~cm}$ apart, feet external rotation $15^{\circ}$, arms along body, eyes focused at eye level target $1.2 \mathrm{~m}$ away, 3 trials of $60 \mathrm{~s}$

Barefoot, feet parallel $8 \mathrm{~cm}$ apart 1 trial of $30 s$ radius, sway density curve

xMean Range of AP AIS \& CON $=31.9 \mathrm{~mm}$ averaged $p=\mathrm{NR}$

\begin{tabular}{|c|c|}
\hline Mean \pm SD Range of ML & $\begin{array}{l}\# \text { AIS }=13 \pm 6.26 \mathrm{~mm}, \# \mathrm{CON}=5.8 \pm \\
6.39 \mathrm{~mm}, p=\mathrm{NR}\end{array}$ \\
\hline Mean \pm SD Range of AP & $\begin{array}{l}\text { \#AIS }=19.5 \pm 5.89 \mathrm{~mm}, \text { \#CON }=10.9 \pm \\
6.1 \mathrm{~mm}, \mathrm{p}=\mathrm{NR}\end{array}$ \\
\hline $\begin{array}{l}\text { Mean } \pm \text { SD Position of } M L \text {, } \\
\text { right displacement }\end{array}$ & $\begin{array}{l}\mathrm{AIS}=5.3 \pm 14.2 \mathrm{~mm}, \mathrm{CON}=3.2 \pm \\
9.3 \mathrm{~mm}, p=\mathrm{NS}\end{array}$ \\
\hline $\begin{array}{l}\text { Mean } \pm \text { SD Position of AP, } \\
\text { posterior displacement }\end{array}$ & $\begin{array}{l}\mathrm{AIS}=26.1 \pm 13.3 \mathrm{~mm}, \mathrm{CON}=36.6 \pm \\
12.4 \mathrm{~mm}, p=0.002^{*}\end{array}$ \\
\hline Mean \pm SD Sway Area & $\begin{array}{l}\# A I S=275 \pm 175 \mathrm{~mm}^{2}, C O N=183 \pm \\
111 \mathrm{~mm}^{2}, p=0.010^{*}\end{array}$ \\
\hline $\begin{array}{l}\times \text { RMS Amplitude, horizontal } \\
\text { motion of ML }\end{array}$ & $\begin{array}{l}\# \text { AIS }=0.5 \pm 0.5 \mathrm{~mm}, \# \mathrm{CON}=0.2 \pm \\
01 \mathrm{~mm}, p<0.05^{*}\end{array}$ \\
\hline $\begin{array}{l}\times \text { RMS Amplitude, horizontal } \\
\text { motion of AP }\end{array}$ & $\begin{array}{l}\# \mathrm{AIS}=0.5 \pm .2 \mathrm{~mm}, \# \mathrm{CON}=0.7 \pm \\
0.4 \mathrm{~mm}, p=\mathrm{NS}\end{array}$ \\
\hline $\begin{array}{l}\text { Mean } \pm \text { SD Position of } \mathrm{ML} \\
\text { right displacement }\end{array}$ & $\begin{array}{l}\mathrm{AIS}=6.5 \pm 10.1 \mathrm{~mm}, \mathrm{CON}=4.4 \pm \\
9.1 \mathrm{~mm}, p=\mathrm{NS}\end{array}$ \\
\hline $\begin{array}{l}\text { Mean } \pm \text { SD Position of AP, } \\
\text { posterior displacement }\end{array}$ & $\begin{array}{l}\mathrm{AIS}=72.7 \pm 12.4 \mathrm{~mm}, \mathrm{CON}=85.0 \pm \\
12.0 \mathrm{~mm}, p=0.043^{*}\end{array}$ \\
\hline Mean \pm SD Sway Area & $\begin{array}{l}\text { AIS }=274.0 \pm 154.0 \mathrm{~mm}^{2}, \mathrm{CON}=190.3 \pm \\
123.5 \mathrm{~mm}^{2}, p=0.009^{*}\end{array}$ \\
\hline Mean \pm SD Range ML & $\begin{array}{l}\text { AIS }=17.0 \pm 6.8 \mathrm{~mm}, \mathrm{CON}=13.4 \pm \\
5.1 \mathrm{~mm}, p=0.05^{*}\end{array}$ \\
\hline Mean \pm SD Range AP & $\begin{array}{l}\mathrm{AIS}=25.9 \pm 13.2 \mathrm{~mm}, \mathrm{CON}=20.4 \pm \\
4.2 \mathrm{~mm}, p=\mathrm{NS}\end{array}$ \\
\hline Mean \pm SD Sway Area & $\begin{array}{l}\text { AIS }=765 \pm 419 \mathrm{~mm}^{2}, \mathrm{CON}=447 \pm \\
98 \mathrm{~mm}^{2}, p=0.004^{*}\end{array}$ \\
\hline $\begin{array}{l}\text { ange of } M L \\
\text { Position of } M L \text {, }\end{array}$ & $\begin{array}{l}\text { \#AIS }=4.8 \pm 1.38 \mathrm{~mm}, \# C O N=4.4 \pm \\
1.13 \mathrm{~mm}, p<0.05^{*}\end{array}$ \\
\hline
\end{tabular}

Mean \pm SD Range of $M L \quad \# A I S=13 \pm 6.26 \mathrm{~mm}$, \#CON=5.8 \pm

\#AIS $=19.5 \pm 5.89 \mathrm{~mm}$, \#CON $=10.9 \pm$ $9.3 \mathrm{~mm}, p=\mathrm{NS}$ $12.4 \mathrm{~mm}, p=0.002^{*}$

\#AIS $=275 \pm 175 \mathrm{~mm}^{2}, \mathrm{CON}=183 \pm$ \#AIS $=0.5 \pm 0.5 \mathrm{~mm}$ \#CON $=0.2 \pm$ $m, p<0.05^{*}$ $0.4 \mathrm{~mm}, p=\mathrm{NS}$

$\mathrm{AIS}=6.5 \pm 10.1 \mathrm{~mm}, \mathrm{CON}=4.4 \pm$ $\mathrm{AIS}=72.7 \pm 12.4 \mathrm{~mm}, \mathrm{CON}=85.0 \pm$ $12.0 \mathrm{~mm}, p=0.043^{*}$

AIS $=274.0 \pm 154.0 \mathrm{~mm}^{2}, \mathrm{CON}=190.3 \pm$ $123.5 \mathrm{~mm}^{2}, p=0.009^{*}$

$\mathrm{AIS}=17.0 \pm 6.8 \mathrm{~mm}, \mathrm{CON}=13.4 \pm$ AIS $=25.9 \pm 13.2 \mathrm{~mm}, \mathrm{CON}=20.4 \pm$ $98 \mathrm{~mm}^{2}, p=0.004^{*}$

Heels together, feet external rotation $30^{\circ}$, eyes focused

$1.13 \mathrm{~mm}, p<0.05^{*}$
Sahlstrand Force platform et al. 1978 L'Electronique 
Table 7 Description of data extracted from all included articles (Continued)

\begin{tabular}{|c|c|c|c|c|}
\hline $\begin{array}{l}\text { Author } \\
\text { Year }\end{array}$ & Force platform & Study protocol & $\begin{array}{l}\text { COP parameters and direction } \\
\text { of displacement }\end{array}$ & Values for AIS, CON, $p$ value \\
\hline \multirow[t]{3}{*}{ [59] } & \multirow{3}{*}{$\begin{array}{l}\text { Appliquee (Montrouge, } \\
\text { France) }\end{array}$} & \multirow{3}{*}{$\begin{array}{l}\text { at eye level on a } 10 \times 10 \mathrm{~cm} \\
\text { reference square } 5 \mathrm{~m} \text { away, } \\
1 \text { trial of } 2 \text { min with } 2 \text {-min } \\
\text { rest intervals }\end{array}$} & right displacement & Data NR, $p<0.05^{*}$ \\
\hline & & & $\begin{array}{l}\times \mathrm{RMS} \pm \mathrm{SD} \text { Range of AP } \\
\times \text { Mean } \pm \text { SD Position of AP }\end{array}$ & $\begin{array}{l}\text { \#AIS }=6.2 \pm 1.9 \mathrm{~mm}, \# \mathrm{CON}=5.6 \pm \\
2.0 \mathrm{~mm}, p<0.05^{*} \\
\text { Data NR, } p=\mathrm{NR}\end{array}$ \\
\hline & & & $\times$ Mean \pm SD Sway Area & $\begin{array}{l}\text { \#AIS }=107.1 \pm 4.9 \mathrm{~mm}^{2}, \# \mathrm{CON}=78.5 \pm \\
4.9 \mathrm{~mm}^{2}, p<0.05^{*}\end{array}$ \\
\hline
\end{tabular}

AIS adolescent idiopathic scoliosis, $A P$ anteroposterior, $C O N$ typically developed adolescents, $C O P$ centre of pressure, $I Q R$ Inter quartile range, $M L$ mediolateral, $N R$ statistical significance not reported, NS no statistical significant, $O B$ observation group, $P B$ pre-bracing group; $p$ value $<0.05$ denotes statistical significance between groups*; $S D$ standard deviation; vs = compared to; $x=$ excluded from meta-analysis; \# = results have been extracted from graph or figure

divergent measurement units [43, 47, 52, 56], use of two separate force plates $[48,50]$, discrete values and graphs missing [53], due to high severity of scoliosis (mean $69.5^{\circ} \pm 8.78$ ) [44] and procedure with heels together [59] (Fig. 1). Two of the excluded studies reported large ES differences in sway area in the qualitative analysis with evident postural instability for AIS compared to CON $[44,59]$. Remaining 9 studies with a total of 491 participants (222 CON; 269 AIS) were included [42, 45, 46, 49, $51,54,55,57,58]$ with a summary of meta-analysis statistics presented in Table 8.

\section{Sway area}

The overall ES for sway area, Cohen's $d$ (pooled) showed a medium ES 0.65, 95\% CI (0.49-0.63) (Table 8) displayed in Fig. 2. Forest plot of Sway area with Cohen's $d$ pooled effect size 0.65 (CI 0.49-0.63). Cochran's Q 5.93 when compared to a chi-squares critical values table the $\chi^{2}$ of heterogeneity test was NS $\left(\chi^{2}=11.071 ; \mathrm{df}=5 ; p=\right.$ $0.05)$. Indicating that the variation between studies was homogeneous therefore accepting the null hypothesis that all studies are equal have a common ES. Further, the $I^{2}$ statistic was $15.71 \%$ indicating low variability across studies, due to heterogeneity rather than chance. This suggests no observed heterogeneity across studies.

\section{Mediolateral position and range}

Cohen's $d$ (pooled) for MLP1 and MLP2 showed no ES difference but MLR showed large ES difference: MLP1 0.15 , 95\% CI (0.08-0.22); MLP2 0.14, 95\% CI (0.080.19); and MLR 0.94, 95\% CI (0.83-1.04) (Table 8) displayed in Fig. 3. Forest plot of Mediolateral position 1 (MLP1) with Cohen's $d$ pooled effect size 0.15 (CI $0.08-$ $0.22)$, Fig. 4. Forest plot of mediolateral position 2 (MLP2) with Cohen's $d$ pooled effect size 0.14 (CI 0.080.19), Fig. 5. Forest plot of mediolateral range with Cohen's $d$ pooled effect size 0.94 (CI 0.83-1.04). Cochran's Q showed that the variation between studies were homogeneous for each of the parameters MLP1 $1.00\left(\chi^{2}=3.841 ; \mathrm{df}=1 ; p=0.05\right)$, MLP2 $1.48\left(\chi^{2}=5.991\right.$; $\mathrm{df}=2 ; p=0.05)$, and MLR 4.84 $\left(\chi^{2}=11.071 ; \mathrm{df}=5 ; p=\right.$ $0.05)$, consistent with Higgins $I^{2}$ results of low variability,

Table 8 Summary of meta-analysis statistics for selected COP parameters, 95\% confidence interval (CI), heterogeneity assessed with Cochran's Q and Higgin's $P^{2}$

\begin{tabular}{|c|c|c|c|c|}
\hline COP parameters & Cohen's d pooled effect size $(95 \%$ Cl) & Cochran's Q & Higgin's $P^{2} \%$ & Studies $(n=9)$ \\
\hline Sway area & $\begin{array}{l}0.65 \\
(0.49-0.63) \text { Medium }\end{array}$ & 5.93 & 15.71 & 6 \\
\hline MLP1 & $\begin{array}{l}0.15 \\
(0.08-0.22) \text { No difference }\end{array}$ & 1.00 & 6.66 & 2 \\
\hline MLP2 & $\begin{array}{l}0.14 \\
(0.08-0.19) \text { No difference }\end{array}$ & 1.48 & 0.00 & 3 \\
\hline MLR & $\begin{array}{l}0.94 \\
(0.83-1.04) \text { Large }\end{array}$ & 4.84 & 0.00 & 6 \\
\hline APP1 & $\begin{array}{l}0.43 \\
(0.31-0.54) \text { Small }\end{array}$ & 1.00 & 4.42 & 2 \\
\hline APP2 & $\begin{array}{l}0.85 \\
(0.72-0.97) \text { Large }\end{array}$ & 2.04 & 1.74 & 3 \\
\hline APR & $\begin{array}{l}0.98 \\
(0.87-1.09) \text { Large }\end{array}$ & 6.35 & 21.30 & 6 \\
\hline
\end{tabular}

COP parameters: Sway area, Mediolateral position 1 (MLP1), Mediolateral position 2 (MLP2), Mediolateral range (MLR), Anteroposterior position (APP1), Anteroposterior position 2 (APP2) and Anteroposterior range (APR). Cohen's $d$ pooled effect size difference defined as small $d=0.2$, medium $d=0.5$ and large $d=0.8$ 


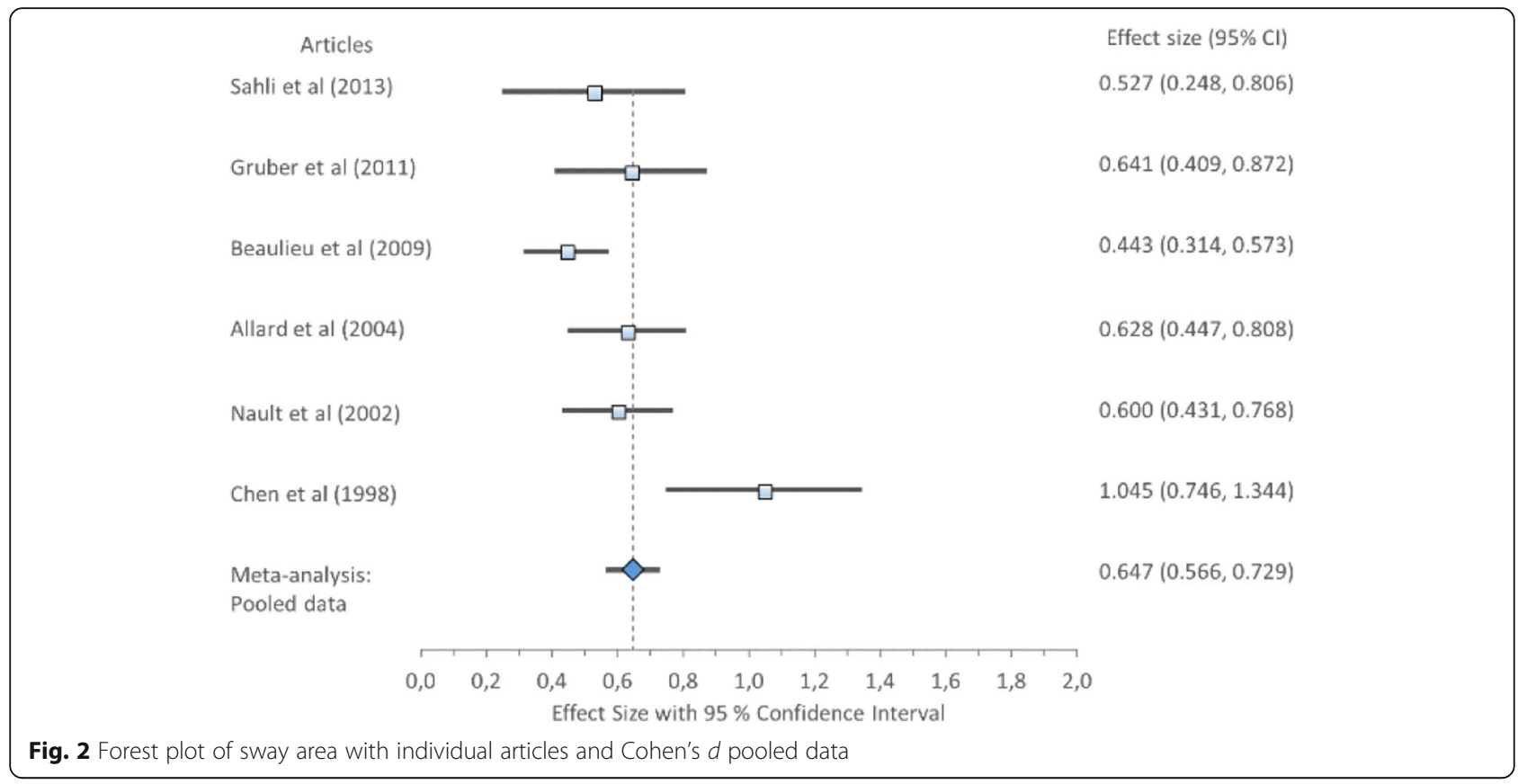

6.66, 0 , and $0 \%$, respectively. This suggests no observed heterogeneity across studies, therefore accepting the null hypothesis that all studies are equal.

\section{Anteroposterior position and range}

Cohen's $d$ (pooled) for APP1 showed small ES difference but a large ES difference for APP2 and APR; APP1 0.43, 95\% CI (0.31-0.54), APP2 0.85, 95\% CI (0.72-0.97) and APR $0.98,95 \%$ CI $(0.87-1.09)$ (Table 8) displayed in Fig. 6. Forest plot of anteroposterior position 1 (APP1) with Cohen's $d$ pooled effect size 0.43 (CI $0.31-0.54$ ), Fig. 7. Forest plot of anteroposterior position 2 (APP2) with Cohen's $d$ pooled effect size 0.85 (CI 0.72-0.97), Fig. 8. Forest plot of anteroposterior range (APR) with Cohen's $d$ pooled effect size 0.98 (CI $0.87-1.09$ ). Cochran's Q showed that the variation between studies were homogeneous for each of the parameters; APP1 $1.00\left(\chi^{2}=3.841 ; \mathrm{df}=1 ; p=0.05\right)$, APP2 $2.04\left(\chi^{2}=5.991\right.$; $\mathrm{df}=2 ; p=0.05)$, and APR $6.35\left(\chi^{2}=11.071 ; \mathrm{df}=5 ; p=\right.$ $0.05)$, consistent with Higgins $I^{2}$ results of low variability across studies: APP1 $4.42 \%$, APP2 $1.74 \%$ and APR $21.30 \%$. This suggests no observed heterogeneity across studies, therefore accepting the null hypothesis that all studies are equal.

\section{Discussion}

This systematic literature review aimed to evaluate the current literature assessing COP parameters in AIS compared with $\mathrm{CON}$ to better understand and determine if AIS affects postural stability. According to the results from 7 different meta-analyses of COP parameters, AIS affects postural stability. In APP2, APR and MLR, a large ES difference was evident while sway area had medium ES difference for AIS compared to CON. This means larger postural instability (range and sway) for AIS with a COP positional shift posteriorly in the sagittal plane.

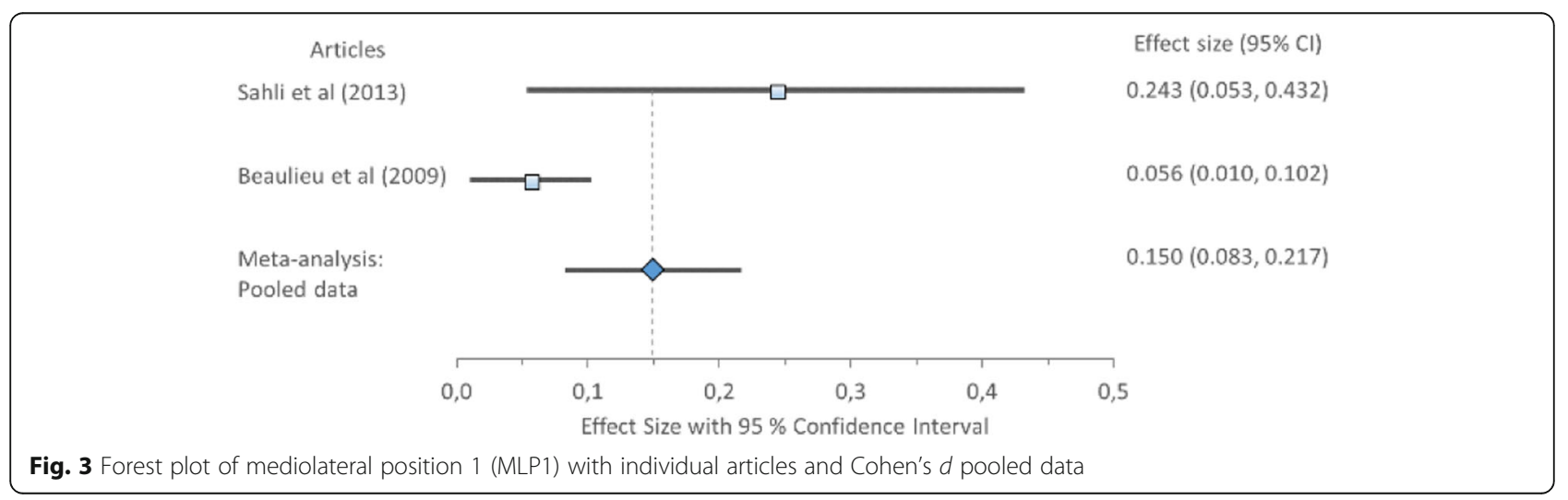




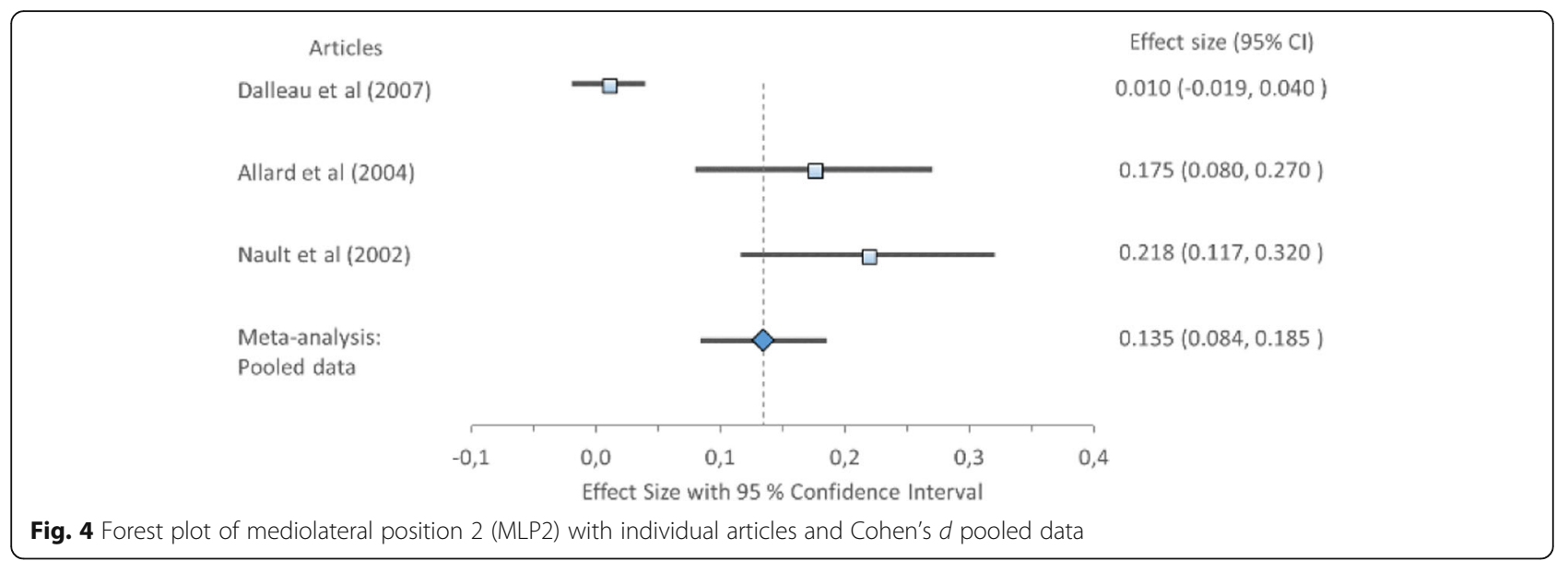

Although AIS is a three-dimensional spinal deformity, the lateral deviation of the spine in the coronal plane is often considered the most distinct deformity. Gruber et al. [46] reasoned that a reduced ML complexity reflected by a larger ML range indicates a more constrained, less adaptable postural control strategy in AIS. The qualitative synthesis showed a positional shift towards right in the frontal plane but also a posterior shift in the sagittal plane in some of the studies. However, when considering the levels of range and position for ML and AP COP parameters in the current review, ML and AP range ES difference was equivalently large. Furthermore, APP1 and APP2 position had greater ES difference compared to MLP1 and MLP2 having no ES difference. Therefore, a shift of COP position posteriorly in the sagittal plane may be considered as a more distinct effect of spinal curvature deformity. Nault et al. [57] investigated the hypothesis that COP parameters are related to standing stability parameters and found that a posterior inclination of the spine was commonly observed in scoliotic subjects. An AP position located closer to the back of the heels has been proposed in previous literature to be influenced by hypokyphotic posture [55]. Recently Leteneur [60] displayed that girls with right thoracic curvature classified as leaning backwards according to trunk sagittal inclination had greater COP range than those leaning forward which is consistent with the findings of present meta-analysis. Biomechanical studies have suggested that the human spine becomes more rotationally unstable with more dorsal shear loads in the thoracolumbar spine with backward trunk sagittal inclination postures [61]. This supports the importance of identifying

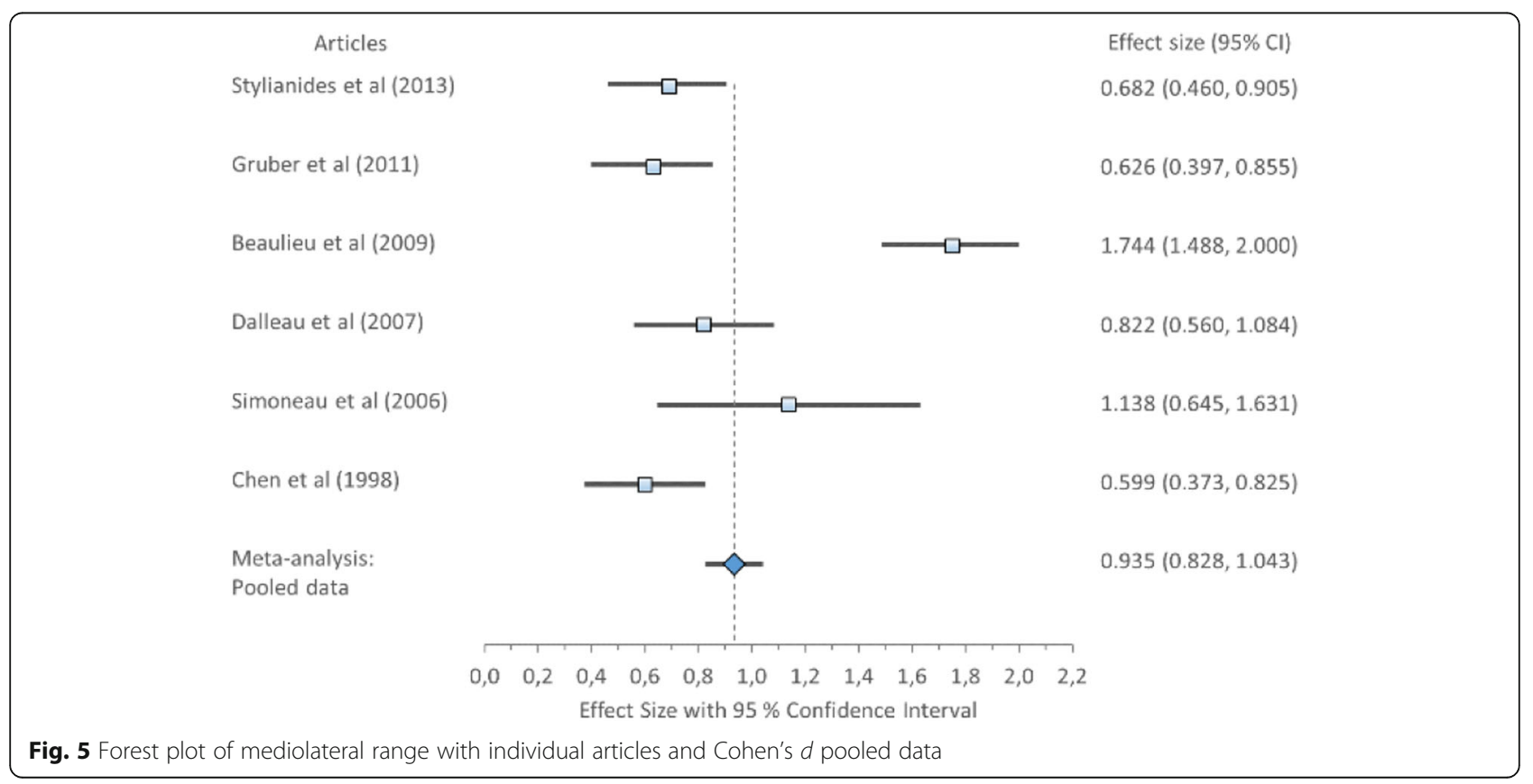




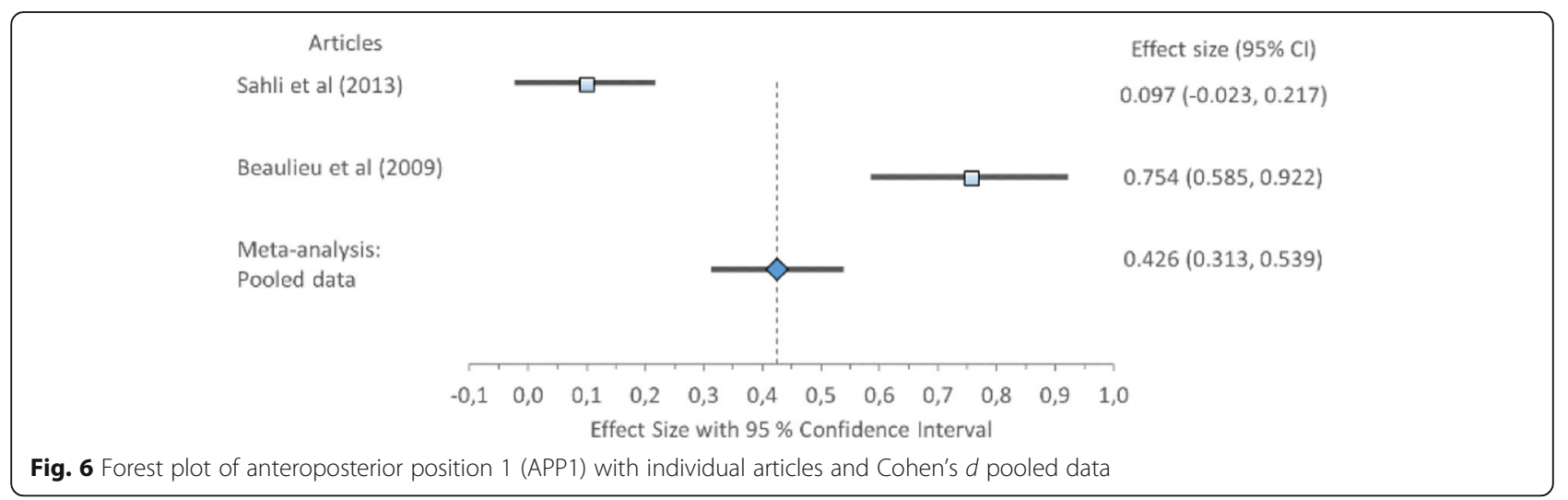

AIS with deficits in postural stability and changed positional shift posteriorly in the sagittal plane and also testing the hypothesis that interventions focusing on scoliosis-specific postural correction as well as postural control may improve postural stability and potentially hinder spinal curvature progression.

An underlying aetiology of AIS is not well established within the current literature, as this condition is thought to be multifactorial in nature and no conclusive evidence exists to support any one theory [56]. Postural instability that presents at the onset of AIS has contributed to a dominant hypothesis that a deficiency in postural control results in scoliosis [15, 18, 62, 63]. Curve type, body segment orientation and body somatotype have all been identified as key factors that may perturb standing balance in the AIS population [55]. Poor postural stability exhibited by AIS patients has been described by two key hypotheses within the literature: a biomechanical and a sensory integration hypothesis. The biomechanical hypothesis gives importance to the biomechanical and morphological changes associated with AIS that are likely to lead to impaired postural stability [42, 64, 65]. These changes include the three-dimensional spinal curvature and deviations in the orientation of the head, shoulders, scapula and pelvis in all three planes [42].

The sensory integration hypothesis indicates individuals with AIS have impaired dynamic regulation of sensorimotor integration due to an inaccurate weighting of sensory inputs. This sensory deprivation has been linked with balance dysfunction reflected in an inability to recalibrate the position of the $\mathrm{COP}$ in relation to the body's COM, and thus, exaggerated body sway oscillations are evident $[15,42]$. A sensory integration disorder may also play an important role in curvature progression due to an inability to readjust COP position to counterbalance COM position over a long time scale [15]. Beaulieu et al. [49] suggest that greater neuromuscular demand is required in AIS to regulate body oscillations due to postural instability. A review studying associated abnormalities found a moderate level of evidence for impaired gait control in AIS [32]. The review summarised that the strength of evidence is low regarding different abnormalities in AIS showing that more research is needed to determine if a consistent pattern of abnormality exists [32]. What clinicians see in their examination of postural control is the net result of disease processes

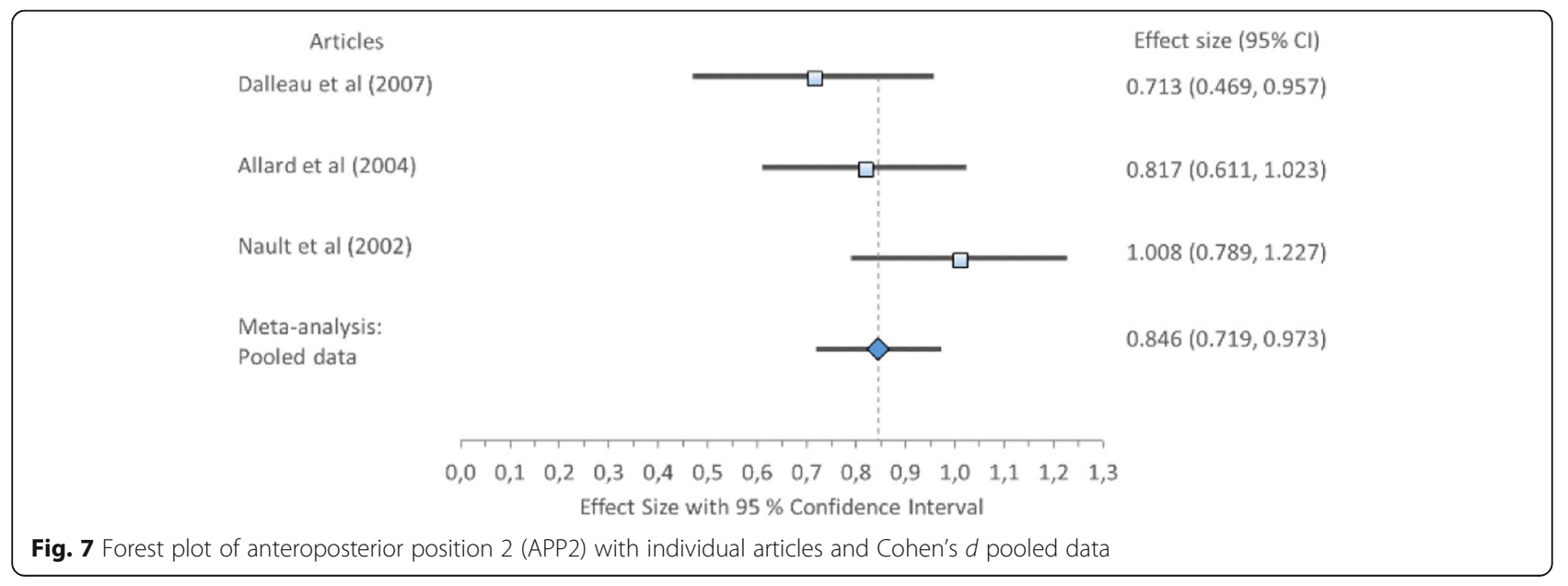




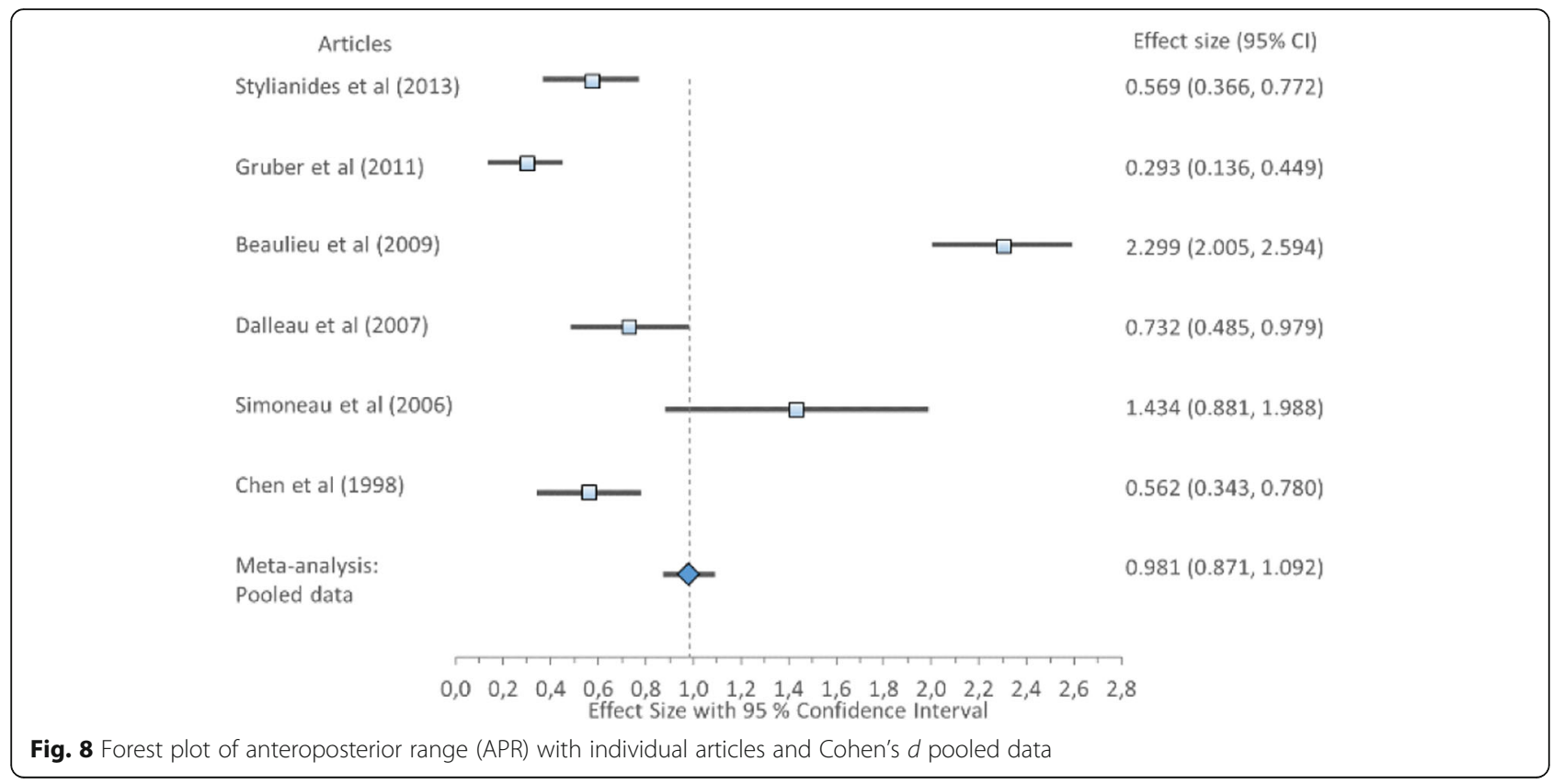

and the patients compensatory strategies in terms of behavioural components and adaptive plasticity in the nervous system [66]. We therefore need to differentiate between primary constraints on balance from compensatory strategies patients use to accomplish the goal of posture. Compensatory strategies may or may not be optimal or effective [67]. The findings of the current study support investigating postural stability and sensory integration in early stage AIS and prospectively to identify cause and effect of the curvature as well as effectiveness of postural control in the prevention of scoliosis progression.

The volume of studies included for analysis was a major strength of the present review. The total number of participants involved provided a greater power to the conclusions drawn from the literature. Furthermore, study participants were representative of the AIS female dominant gender distributions apparent in the community. Comparisons and conclusions are however limited by posturography methodological differences between studies and a need for standardisation of study protocols for future research. A review by Ruhe et al. [29] concluded that a minimum of 90-s sampling time, an average of three to five repetitions and sampling frequency of $100 \mathrm{~Hz}$ are required to reach acceptable reliability for most COP parameters. Therefore, we suggest that future studies assessing COP parameters in AIS adopt the above study protocol as baseline testing in unperturbed stance with the addition of participants' feet spaced $23 \mathrm{~cm}$ apart, pointing externally $15^{\circ}$, and eyes focused on target $1.2 \mathrm{~m}$ ahead at eye level. More studies investigating sensory integration, with perturbed stance, are needed to allow for meta-analysis of data from studies testing proposed sensory integration hypothesis.
It is also important to consider potential limitations of the current review. For example, 2 of the studies in the meta-analyses showed a high risk of bias [54, 57], 5 moderate $[45,46,51,55,58]$ and 2 low risk-of-bias [42, 49]. Furthermore, posturography methodological quality checklist displayed 1 study showing low [58], 3 moderate $[42,46,54]$ and 5 high-quality scores for studies included in the meta-analyses $[45,49,51,55,57]$. Discrepant methodological quality may be explained by differing aims, sampling methods, AIS curve types and size heterogeneity as well as reporting of maturation characteristics. For example, some studies primarily aimed to investigate the effect of back pack load on COP parameters, differences in gait patterns and risk of falls within subgroups of AIS [42, 53, 58]. None of the studies discussed statistical power and there was a lack of consistency in reporting sampling as well as AIS classification. These factors may influence the variation around the COP parameter means within AIS samples, but one must also consider the inherent variation that exists within CON. Future research in this field would benefit from standardised reporting of sampling, posturography methods as well as anthropometrical and maturation characteristics of AIS and typically developed adolescent populations.

\section{Conclusion}

There is moderate quality evidence for decreased postural stability in AIS measured as COP parameters sway area, ML and AP range with a positional shift posteriorly in the sagittal plane. The findings support studying postural stability in early stage AIS and also prospectively 
identify cause and effect of the curvature as well as effectiveness of postural control interventions in the prevention of scoliosis progression.

\begin{abstract}
Abbreviations

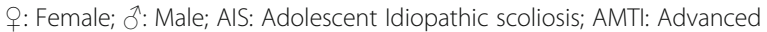
Mechanical Technology Incorporated; AP: Anteroposterior;

APP: Anteroposterior position; APR: Anteroposterior Range; BOS: Base of support; Cl: Confidence interval; CINAHL: The Cumulative Index of Nursing and Allied Health Literature database; COG: Centre of gravity; COM: Centre of body mass; CON: Typically developed adolescents as control group; COP: Centre of pressure; DF: Degrees of freedom; ES: Effect size; GRF: Ground reaction forces; Hz: Herz, unit of frequency defined as 1 cycle per second; ML: Mediolateral; MLP: Mediolateral position; MLR: Mediolateral range; NR: Not reported; NS: No statistical significance; OB: Observation groups; PB: Pre-bracing; PEDro: Physiotherapy Evidence Database; PRISMA: The Preferred Reporting Items for Systematic Reviews and Meta-Analyses guidelines; PubMed: Publishers Medline by the National Library of Medicine journal citation database; S: Statistical significance; Scopus: Abstract and indexing database by Elsevier; SD: Standard deviation; SE: Standard error; SEM: Standard error of measurements
\end{abstract}

\section{Acknowledgements}

Henrik Hedevik, statistician at Linkopings University, has assisted in the metaanalysis process and presentations of data.

\section{Availability of data and materials}

The Excel spreadsheet used for meta-analysis is available from the corresponding author on reasonable request.

\section{Declarations}

The research described has not yet been published, submitted or accepted for publication.

\section{Authors' contributions}

All authors were involved in the conception of the study and design of the study and helped to draft the manuscript. MD, FA and IR were involved with the database searches and record screening. MD, FA, IR and AA were involved in the study review process and data analyses. All authors read and approved the final manuscript.

\section{Ethics approval and consent to participate}

Not applicable

\section{Consent for publication}

Not applicable

\section{Competing interests}

The authors declare that they have no competing interests.

\section{Publisher's Note}

Springer Nature remains neutral with regard to jurisdictional claims in published maps and institutional affiliations.

\section{Author details}

'Department of Medical and Health Sciences, Division of Physiotherapy, Linkoping University, 58183 Linkoping, Sweden. ${ }^{2}$ Department of Clinical and Rehabilitation Services, Faculty of Health Science and Medicine, Bond Institute of Health and Sport, Bond University, 2 Promethean Way, Robina, QLD 4226, Australia.

Received: 28 May 2018 Accepted: 11 July 2018

Published online: 03 September 2018

\section{References}

1. Shumway-Cook A, Woollacott MH. A conceptual framework for clinical practice \& normal postural control. In: Shumway-Cook A, Woollacott MH, editors. Motor control: translating research into clinical practice. Philadelphia: Wolters Kluwer; 2017. p.141-193.

2. Massion J. Postural control system. Curr Opin Neurobiol. 1994;4:877-87.
3. Latash M, Hadders-Algra M. What is posture and how is it controlled? In: Hadders-Algra M, Brogren Carlberg E, editors. Postural control: a key issue in developmental disorders. London: Mac Keith Press; 2008. p. 3-21.

4. Horak FB, Macpherson JM. Postural Orientation and Equilibrium. Compr Physiol. 2011; Suppl 29: 255-292. Handbook of Physiology, Exercise: Regulation and Integration of Multiple Systems: First published in print 1996; https://doi.org/10.1002/cphy.cp120107.

5. Winter DA. Kinetics: forces and moments of force. Biomechanics \& motor control of human movement. USA: John Wiley \& Sons Inc; 2009. p.10-11, 26-43, 85-87,107-127, 130-137, 281-289.

6. Bottaro A, Casadio M, Morasso PG, Sanguineti V. Body sway during quiet standing: is it the residual chattering of an intermittent stabilization process? Hum Mov Sci. 2005;24:588-615.

7. Horak FB. Postural orientation and equilibrium: what do we need to know about neural control of balance to prevent falls? Age Ageing. 2006; https:// doi.org/10.1093/ageing/afl077.

8. Schlösser TPC, Colo D, Castelein RM. Etiology and pathogenesis of adolescent idiopathic scoliosis. Semin Spine Surg. 2015;27:2-8.

9. Rigo M. Patient evaluation in idiopathic scoliosis: radiographic assessment, trunk deformity and back asymmetry. Physiotherapy Theory And Practice. 2011;27:7-25.

10. Ekinci S, Ersen O. Adolescent idiopathic scoliosis. Arch Clin Exp Surg. 2014;3:174-82.

11. Weinstein SL. Adolescent idiopathic scoliosis. Prevalence and natural history Orthopäde. 1989;18:74-86.

12. Konieczny M, Senyurt H, Krauspe R. Epidemiology of adolescent idiopathic scoliosis. J Child Orthop. 2013;7:3.

13. Choudhry MN, Ahmad Z, Verma R. Adolescent idiopathic scoliosis. The Open Orthopaedics Journal. 2016; https://doi.org/10.2174/ 1874325001610010143.

14. Weinstein SL, Dolan LA, Cheng JC, Danielsson A, Morcuende JA. Adolescent idiopathic scoliosis. Lancet. 2008;371:1527-37.

15. Herman R, Mixon J, Fisher A, Maulucci R, Stuyck J. Idiopathic scoliosis and the central nervous system: a motor control problem. The Harrington lecture, 1983. Scoliosis Research Society. Spine. 1985;10:1-14.

16. Catanzariti JF, Agnani O, Guyot MA, Wlodyka-Demaille S, Khenioui H, Donze C. Does adolescent idiopathic scoliosis relate to vestibular disorders? A systematic review. Annals of Physical and Rehabilitation Medicine. 2014;57:465-79.

17. Burwell RG, Freeman BJ, Dangerfield PH, Aujla RK, Cole AA, Kirby A, et al. Etiologic theories of idiopathic scoliosis: neurodevelopmental concept of maturational delay of the CNS body schema ("body-in-the-brain"). Stud Health Technol Inform 2006;123:72-79.

18. Burwell RG, Aujla RK, Grevitt MP, Dangerfield PH, Moulton A, Randell TL, et al. Pathogenesis of adolescent idiopathic scoliosis in girls - a double neuroosseous theory involving disharmony between two nervous systems, somatic and autonomic expressed in the spine and trunk: possible dependency on sympathetic nervous system and hormones with implications for medical therapy. Scoliosis 2009; https://doi.org/10.1186/ 1748-7161-4-24

19. Byl NN, Holland S, Jurek A, Hu SS. Postural imbalance and vibratory sensitivity in patients with idiopathic scoliosis: implications for treatment. J Orthop Sports Phys Ther. 1997;26:60-8.

20. Mirovsky Y, Blankstein A, Shlamkovitch N. Postural control in patients with severe idiopathic scoliosis: a prospective study. J Pediatr Orthop B. 2006;15:168-71.

21. Karimi MT, Kavyani M, Kamali M. Balance and gait performance of scoliotic subjects: a review of the literature. J Back Musculoskelet Rehabil. 2016;29:403-15.

22. Paillard T, Noé F. Techniques and methods for testing the postural function in healthy and pathological subjects. Biomed Res Int. 2015; https://doi.org/ 10.1155/2015/891390.

23. Chaudhry H, Findley T, Quigley KS, Bukiet B, Ji Z, Sims T, et al. Measures of postural stability. JRRD 2004;41:713-720.

24. Prieto TE, Myklebust JB, Hoffmann RG, Lovett EG, Myklebust BM. Measures of postural steadiness: differences between healthy young and elderly adults. IEEE Trans Biomed Eng. 1996;43:956-66.

25. Wolff DR, Rose J, Jones VK, Bloch DA, Oehlert JW, Gamble JG. Postural balance measurements for children and adolescents. J Orthop Res. 1998;16:271-5.

26. Palmieri RM, Ingersoll CD, Stone MB, Krause BA. Center-of-pressure parameters used in the assessment of postural control. J Sport Rehabil. 2002;11:51-66. 
27. Gauchard GC, Lascombes P, Kuhnast M, Perrin PP. Influence of different types of progressive idiopathic scoliosis on static and dynamic postural control. Spine. 2001;26:1052-8.

28. Enoka RM. Neuromechanics of human movement. Champaign IL: Human Kinetics; 2015. p. 3-55, 255-307, 448-49.

29. Ruhe A, Fejer R, Walker B. The test-retest reliability of centre of pressure measures in bipedal static task conditions - a systematic review of the literature. Gait \& Posture. 2010;32:436-45.

30. Lin D, Seol H, Nussbaum MA, Madigan ML. Reliability of COP-based postural sway measures and age-related differences. Gait \& Posture. 2008;28:337-42.

31. Liberati R, Altman DG, Tetzlaff J, Mulrow C, Gøtzsche PC, loannidis JPA. The PRISMA statement for reporting systematic reviews and meta-analyses of studies that evaluate health care interventions: explanation and elaboration. PLoS Med. 2009; https://doi.org/10.1371/journal.pmed.1000100.

32. Schlösser TPC, van der Heijden GJMG, Versteeg AL, Castelein RM. How 'idiopathic' is adolescent idiopathic scoliosis? A systematic review on associated abnormalities. PLoS One. 2014; https://doi.org/10.1371/journal. pone.0097461

33. Mazaheri M, Coenen P, Parnianpour M, Kiers H, Review v DJH. Low back pain and postural sway during quiet standing with and without sensory manipulation: a systematic review. Gait \& Posture. 2013; https://doi.org/10. 1016/j.gaitpost.2012.06.013.

34. Cohen J. Statistical power analysis for the behavioral sciences. 3rd ed. Hillsdale: L. Erlbaum Associates; 1988.

35. Becker LA. Effect size calculators. University of Colorado Colorado Springs. 1999; http://www.uccs.edu/ Ibecker/. Accessed 14 March 2017

36. StatsToDo. StatTools, Statistics Toolkit (STATTOOLS). The Chinese University of Hong Kong, Department of Obstetrics and Gynaecology. 1995. Means and SDs Into One Group Program. https://www.statstodo.com/ CombineMeansSDs_Pgm.php/Combine. Accessed 17 Nov 2017.

37. Neyeloff JL, Fuchs SC, Moreira LB. Meta-analyses and Forest plots using a microsoft excel spreadsheet: step-by-step guide focusing on descriptive data analysis. BMC Research Notes. 2012;5:52.

38. Higgins JPT, Thompson G, Deeks JJ, Altman DG. Measuring inconsistency in meta-analyses. BMJ. 2003;327:557-60.

39. Lakens D. Calculating and reporting effect sizes to facilitate cumulative science: a practical primer for t-tests and ANOVAs. Front Psychol. 2013; https://doi.org/10.3389/fpsyg.2013.00863.

40. Hesser H, Andersson G. Introduktion till metaanalys och systematiska översikter. 1st ed. Studentlitteratur: Lund; 2015

41. Huedo-Medina TB, Sanchez-Meca J, Marin-Martinez F, Botella J. Assessing heterogeneity in meta-analysis: Q statistic or 12 index? Psychol Methods. 2006;11:193-206.

42. Sahli S, Rebai H, Ghroubi S, Yahia A, Guermazi M, Study EMHC. The effects of backpack load and carrying method on the balance of adolescent idiopathic scoliosis subjects. Spine J. 2013;13:1835-42.

43. Park JY, Park GD, Lee SG, Lee JC. The effect of scoliosis angle on center of gravity sway. J Phys Ther Sci. 2013;25:1629-31.

44. De Santiago HAR, Reis JG, Gomes MM, Da Silva Herrero CFP, Defino HLA, De Abreu DCC. The influence of vision and support base on balance during quiet standing in patients with adolescent idiopathic scoliosis before and after posterior spinal fusion. Spine J. 2013;13:1470-6.

45. Stylianides GA, Dalleau G, Begon M, Rivard CH, Allard P. Pelvic morphology, body posture and standing balance characteristics of adolescent ablebodied and idiopathic scoliosis girls. PLoS One. 2013; https://doi.org/10. 1371/journal.pone.0070205.

46. Gruber AH, Busa MA, Gorton GE, Van Emmerik REA, Masso PD, Hamill J. Time-to-contact and multiscale entropy identify differences in postural control in adolescent idiopathic scoliosis. Gait \& Posture. 2011;34:13-8.

47. Dalleau G, Dalleau G, Damavandi M, Leroyer P, Verkindt C, Rivard CH, Allard $P$. Horizontal body and trunk center of mass offset and standing balance in scoliotic girls. Eur Spine J. 2011;20:123-8.

48. Valles KDB, Long JT, Riedel SA, Graf A, Krzak J, Hassani S, et al. Using a biplanar postural stability model to assess children with scoliosis. 2009; https://doi.org/10.1109/IEMBS.2009.5333839.

49. Beaulieu M, Toulotte C, Gatto L, Rivard CH, Teasdale N, Simoneau M, Allard P. Postural imbalance in non-treated adolescent idiopathic scoliosis at different periods of progression. Eur Spine J. 2009;18:38-44.

50. Zabjek KF, Coillard C, Rivard CH, Prince F. Estimation of the centre of mass for the study of postural control in idiopathic scoliosis patients: a comparison of two techniques. Eur Spine J. 2008;17:355-60.
51. Dalleau G, Allard MS, Beaulieu M, Rivard CH, Allard P. Free moment contribution to quiet standing in able-bodied and scoliotic girls. Eur Spine J. 2007;16:1593-9.

52. Simoneau M, Mercier P, Blouin J, Allard P, Teasdale N. Altered sensoryweighting mechanisms is observed in adolescents with idiopathic scoliosis. BMC Neurosci. 2006a;7:68.

53. Chow DHK, Kwok MLY, Cheng JCY, Lao MLM, Holmes AD, Au-Yang A. The effect of backpack weight on the standing posture and balance of schoolgirls with adolescent idiopathic scoliosis and normal controls. Gait \& Posture. 2006;(2):173-81.

54. Simoneau M, Richer N, Mercier P, Allard P, Teasdale N. Sensory deprivation and balance control in idiopathic scoliosis adolescent. Exp Brain Res. 2006b; https://doi.org/10.1007/s00221-005-0246-0.

55. Allard P, Chavet $P$, Barbier F, Gatto L, Labelle $H$, Sadeghi $H$. Effect of body morphology on standing balance in adolescent idiopathic scoliosis. Am J Phys Med Rehabil. 2004;83:689-97.

56. Silferi $\mathrm{V}$, Rougier $\mathrm{P}$, Labelle $\mathrm{H}$, Allard P. Postural control in idiopathic scoliosis: comparison between healthy and scoliotic subjects. Rev Chir Orthop Reparatrice Appar Mot. 2004;90:215-25.

57. Nault ML, Allard P, Hinse S, Le Blanc R, Caron O, Labelle H, et al. Relations between standing stability and body posture parameters in adolescent idiopathic scoliosis. Spine 2002:27: 1911-1917.

58. Chen PQ, Wang JL, Tsuang YH, Liao TL, Huang PI, Hang YS. The postura stability control and gait pattern of idiopathic scoliosis adolescents. Clin Biomech. 1998;13(Suppl 1):52-8.

59. Sahlstrand T, Ortengren R, Nachemson A. Postural equilibrium in adolescent idiopathic scoliosis. Acta Orthop Scand. 1978;49:354-65.

60. Leteneur S, Simoneau-Buessinger E, Barbier F, Rivard CH, Allard P. Effect of natural sagittal trunk lean on standing balance in untreated scoliotic girls. Clin Biomech (Bristol, Avon). 2017;49:107-12.

61. Kouwenhoven JWM, Castelein RM, Smit TH, Van Der Veen AJ, Kingma I, Van Dieën JH. Effects of dorsal versus ventral shear loads on the rotational stability of the thoracic spine: a biomechanical porcine and human cadaveric study. Spine. 2007;32(23):2545-50

62. Sahlstrand T, Sellden U. Nerve conduction velocity in patients with adolescent idiopathic scoliosis. Scand J Rehabil Med. 1980;12:25-6.

63. Yamamoto $\mathrm{H}$, Yamada K. Equilibrial approach to scoliotic posture. Agressologie. 1976;17:61-5.

64. Sawatzky B, Tredwell S, Sanderson D. Postural control and trunk imbalance following Cotrel-Dubousset instrumentation for adolescent idiopathic scoliosis. Gait \& Posture. 1997;5:116-9.

65. Masso PD, Gorton GE. Quantifying changes in standing body segment alignment following spinal instrumentation and fusion in idiopathic scoliosis using an optoelectronic measurement system. Spine. 2000;25:457-62.

66. Visser JE, Carpenter MG, van der Kooij H, Bloem BR. The clinical utility of posturography. Clin Neurophysiol. 2008;119:2424-36.

67. Horak FB. Clinical assessment of balance disorders. Gait \& Posture. 1997; 6:76-84.

Ready to submit your research? Choose BMC and benefit from:

- fast, convenient online submission

- thorough peer review by experienced researchers in your field

- rapid publication on acceptance

- support for research data, including large and complex data types

- gold Open Access which fosters wider collaboration and increased citations

- maximum visibility for your research: over $100 \mathrm{M}$ website views per year

At $\mathrm{BMC}$, research is always in progress.

Learn more biomedcentral.com/submissions 